\title{
$\beta$-Cyclodextrin- and adamantyl-substituted poly(acrylate) self-assembling aqueous networks designed for controlled complexation and release of small molecules
}

\author{
Liang Yan ${ }^{1}$, Duc-Truc Pham ${ }^{1}$, Philip Clements ${ }^{1}$, Stephen F. Lincoln*1, Jie Wang², \\ Xuhong Guo ${ }^{*}$ and Christopher J. Easton ${ }^{3}$
}

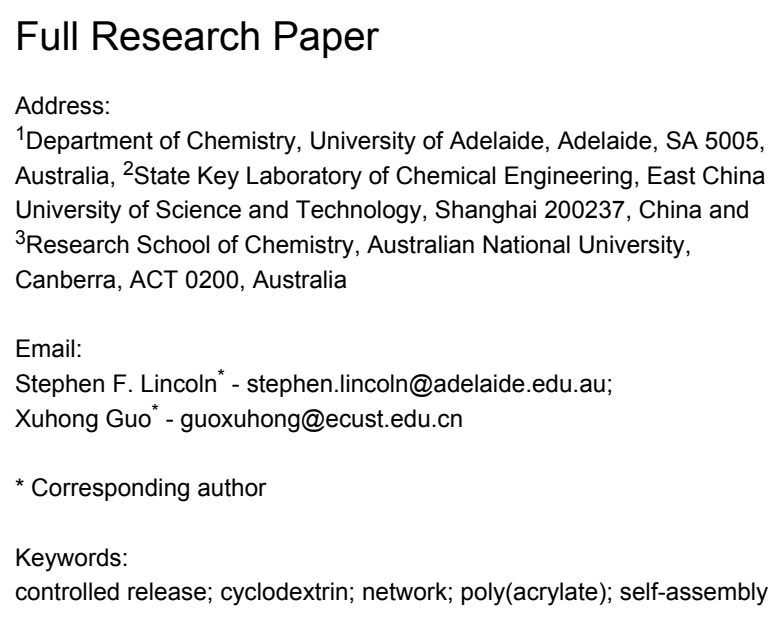

\begin{abstract}
Three aqueous self-assembling poly(acrylate) networks have been designed to gain insight into the factors controlling the complexation and release of small molecules within them. These networks are formed between $8.8 \% 6^{\mathrm{A}}-\left(2\right.$-aminoethyl)amino- $6^{\mathrm{A}}-\mathrm{deoxy}-$ $6^{\mathrm{A}}-\beta$-cyclodextrin, $\beta$-CDen, randomly substituted poly(acrylate), PAA $\beta$-CDen, and one of the 3.3\% 1-(2-aminoethyl)amidoadamantyl, ADen, 3.0\% 1-(6-aminohexyl)amidoadamantyl, ADhn, or 2.9\% 1-(12-aminododecyl)amidoadamantyl, ADddn, randomly substituted poly(acrylate)s, PAAADen, PAAADhn and PAAADddn, respectively, such that the ratio of $\beta$-CDen to adamantyl substituents is ca. 3:1. The variation of the characteristics of the complexation of the dyes methyl red, methyl orange and ethyl orange in these three networks and by $\beta$-cyclodextrin, $\beta-\mathrm{CD}$, and PAA $\beta$-CDen alone provides insight into the factors affecting dye complexation. The rates of release of the dyes through a dialysis membrane from the three aqueous networks show a high dependence on host-guest complexation between the $\beta$-CDen substituents and the dyes as well as the structure and the viscosity of the network as shown by ITC, ${ }^{1} \mathrm{H}$ NMR and UV-vis spectroscopy, and rheological studies. Such networks potentially form a basis for the design of controlled drug release systems.
\end{abstract}

\section{Introduction}

The formation of self-assembling aqueous polymer networks through the complexation of hydrophobic polymer substituents by cyclodextrin oligomers [1-4] and cyclodextrin substituted polymers [5-19] to form cross-links between polymer strands is well-established. Depending upon their composition, these

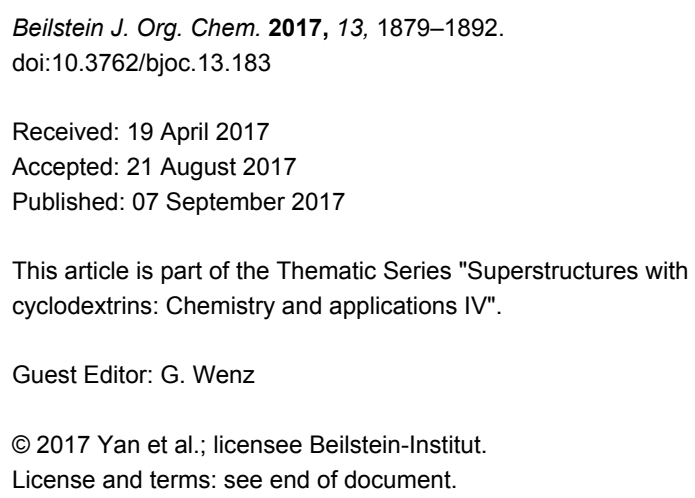


lated by either $\mathrm{pH}$ variation [28,30,34] or light irradiation $[38,45]$. The drug types include small molecular species, exemplified by diflunisal, fluconazole [40] and curcumin [37], along with larger species exemplified by RNA and DNA segments $[26,32,33,36,39,47]$. Some systems are designed to target specific tissues [26,35].

We are particularly interested in the extent to which small molecule guest complexation and release characteristics may be designed into the structure of aqueous networks formed between a $\beta$-cyclodextrin-substituted poly(acrylate) and three adamantylsubstituted poly(acrylate)s. Accordingly, we report an ITC, ${ }^{1} \mathrm{H}$ NMR and UV-vis spectroscopic and rheological study of three self-assembling networks formed between the $8.8 \%$ $6^{\mathrm{A}}$-(2-aminoethyl)amino- $6^{\mathrm{A}}$-deoxy- $6^{\mathrm{A}}-\beta$-cyclodextrin, $\beta$-CDen, randomly substituted poly(acrylate), PAA $\beta$-CDen [13], and 3.3\% 1-(2-aminoethyl)amido-adamantyl, ADen, 3.0\% 1-(6aminohexyl)amidoadamantyl, ADhn, or 2.9\% 1-(12-aminododecyl)amidoadamantyl, ADddn, randomly substituted poly(acrylate)s, PAAADen [11], PAAADhn [15] and PAAADddn [15], respectively, where the poly(acrylate) is of $250 \mathrm{kDa}$ average molecular weight prior to substitution (Figure 1). The network formation is driven by the $\beta$-CDen substituents complexing the adamantyl substituents, ADen, ADhn or ADddn, to form cross-links between the PAA $\beta$-CDen strands and the PAAADen, PAAADhn or PAAADddn strands. The adamantyl group is selected as the guest substituent as it is strongly complexed by $\beta-\mathrm{CD}[48], \beta-\mathrm{CD}$ oligomers $[3,4]$ and $\beta$-CD-substituted polymers $[10,14,16]$, and drives the selfassembly of aqueous chitosan [1,5,9], hyaluronic acid [8,9] and poly(acrylate) networks $[14,15,19]$. In aqueous solutions equimolar in PAA $\beta$-CDen strands and PAAADen, PAAADhn or PAAADddn strands, the concentration of the $\beta$-CDen substituents is in ca. three-fold excess over that of the adamantyl substituents as a consequence of the ca. three-fold greater percentage substitution of PAA $\beta$-CDen. Thus, when host-guest complexation between the poly(acrylate) substituents of the network is complete, ca. two thirds of the $\beta$-CDen substituents remain available to complex other hydrophobic species exemplified by the dyes methyl red, MR, methyl orange, MO, and ethyl orange, EO, chosen for this study (Figure 1).

\section{Results and Discussion Isothermal titration calorimetry (ITC) studies of substituted poly(acrylate) network formation}

In aqueous solution, the host $\beta$-CDen substituents of PAA $\beta$ CDen complex the guest adamantyl substituents of PAAADen, PAAADhn and PAAADddn to form poly(acrylate) networks as exemplified by the PAA $\beta$-CDen/PAAADen system according

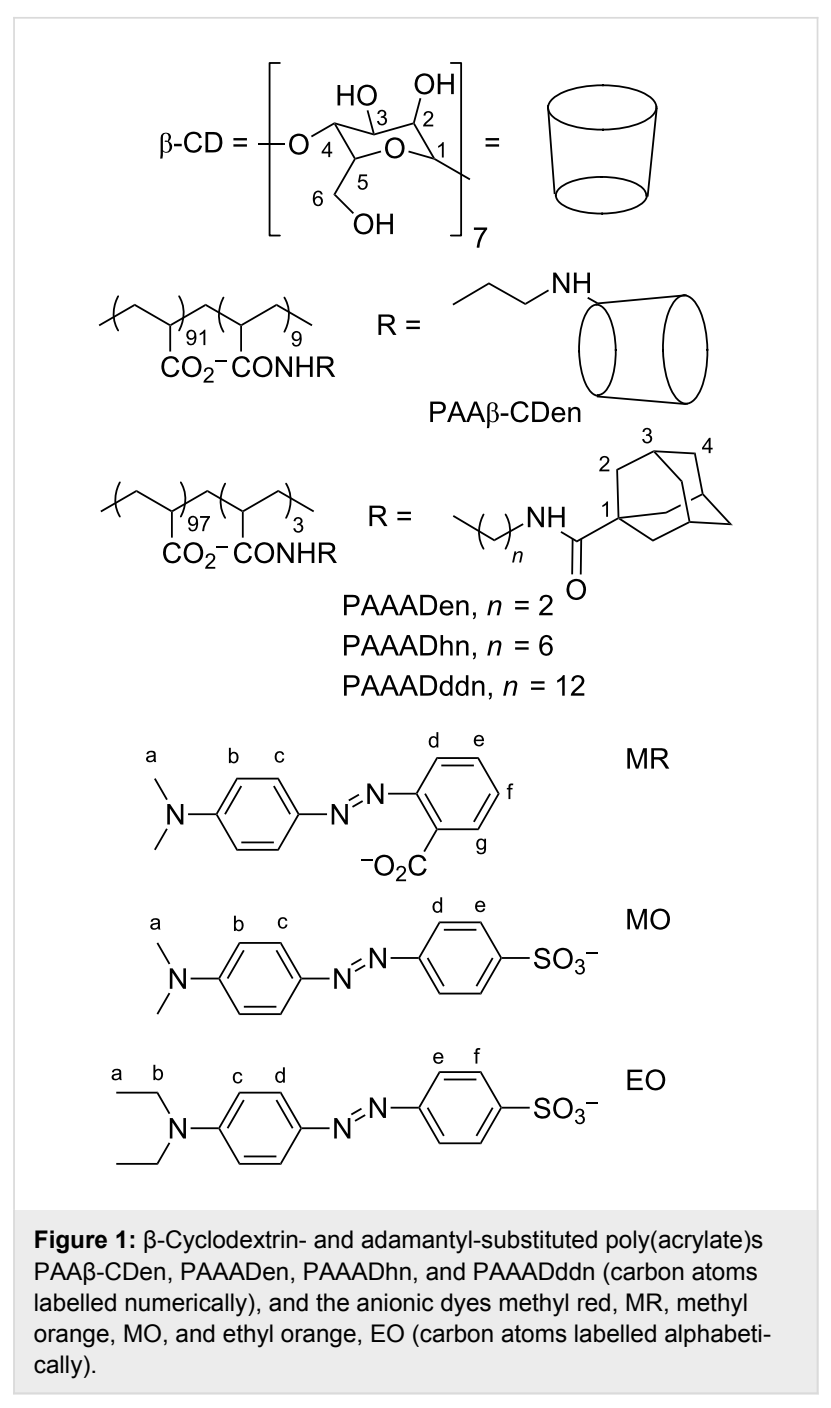

to Equation 1. The complexation constant, $K$, for the host-guest complexation between the $\beta$-CDen and ADen substituents within the network is given by Equation 2. The data for the titration of a PAA $\beta$-CDen solution into a PAAADen, a PAAADhn, or a PAAADddn solution together with the best-fit of an algorithm for a single complexation (Equation 1) for the PAA $\beta$-CDen/PAAADen system (and analogous equations for the other two systems) to these data appear in Figure 2 and Figure 3 and Figure S1 in Supporting Information File 1. The derived $K$ and the corresponding $\Delta H, T \Delta S$, and $N$ values are given in Table 1. The ratio of the number of ADen substituents of PAAADen complexed by a single $\beta$-CDen substituent of PAA $\beta$-CDen, $N$, is expected to be unity for unhindered $1: 1$ complexation and corresponds to the ratio of complexed $\beta$-CDen substituents to complexed adamantyl substituents at the inflexion point of the ITC titration curve (Equation 3). However, the $N=0.78$ and 0.87 derived for each $\beta$-CDen substituent complexing either a ADen or ADhn substituent, respectively, are less than unity as also observed in other studies $[1,3-5,8]$. 
(a)

Time / min

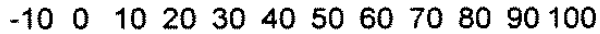

(b)

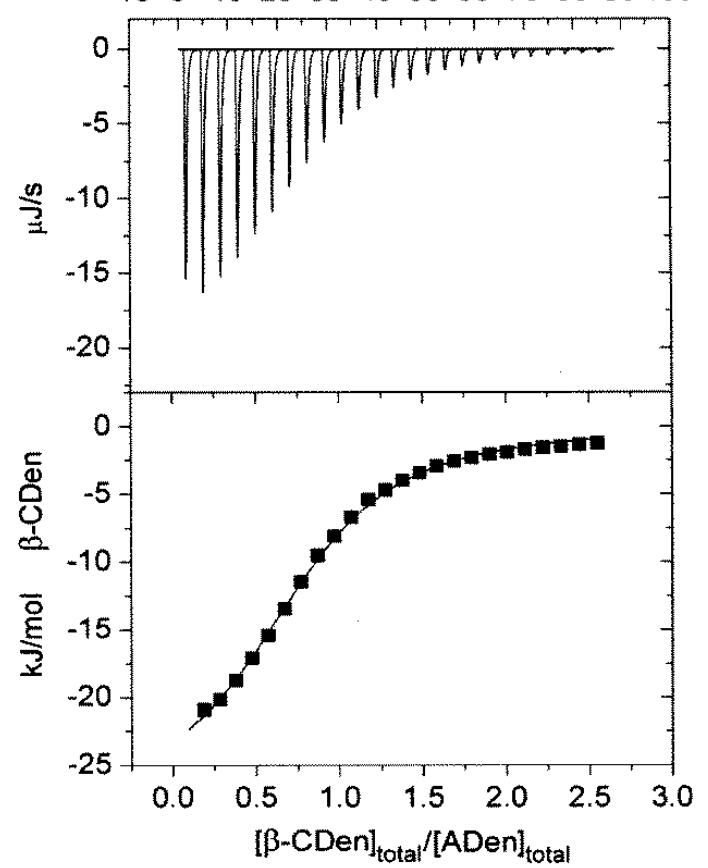

(c)

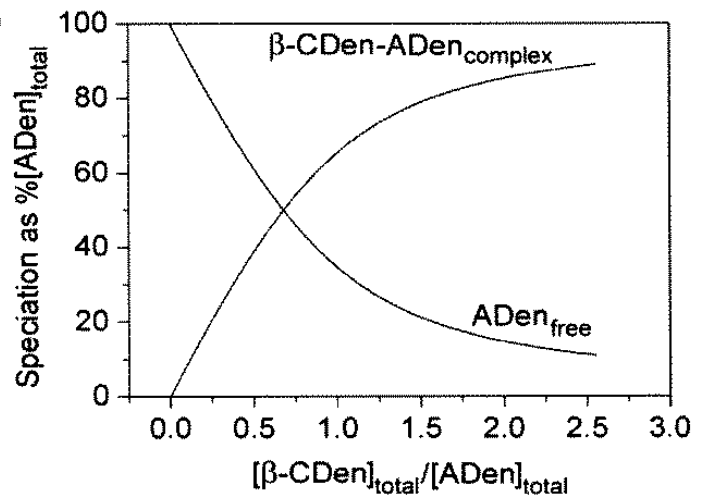

Figure 2: ITC data for the PAA -CDen/PAAADen, system obtained in aqueous $\mathrm{Na}_{2} \mathrm{HPO}_{4} / \mathrm{KH}_{2} \mathrm{PO}_{4} \mathrm{pH} 7.0$ buffer at $I=0.10 \mathrm{~mol} \mathrm{dm}^{-3}$. (a) Titration of $10 \mathrm{~mm}^{3}$ aliquots of $0.62 \mathrm{wt} \%$ PAA $\beta$-CDen ([B-CDen] $\left.=2.84 \times 10^{-3} \mathrm{~mol} \mathrm{dm}^{-3}\right)$ into $1.46 \mathrm{~cm}^{3}$ of $0.062 \mathrm{wt} \%$ PAAADen ([ADen] $=2.06 \times 10^{-4} \mathrm{~mol} \mathrm{dm}^{-3}$ ). (b) The solid curve shows the best fit of an algorithm for host-guest complexation between the $\beta$-CDen and ADen substituents to the titration data points.

(c) Speciation plots showing for the variation of the

$[\beta-C D e n-A D e n]_{c o m p l e x}$ and of the $[A D e n]_{\text {free }}$ as percentage $a$ of $[A \text { Den }]_{\text {total }}=100 \%$.

This is attributed to either steric hindrance by the poly(acrylate) backbone, or hydrophobic association of the adamantyl substituents, or both, hindering complexation.

$$
\beta \text {-CDen + ADen } \stackrel{K}{\rightleftharpoons} \beta \text {-CDen-ADen }
$$

$$
\begin{aligned}
& K=[\beta-\text { CDen-ADen }] /([\beta-\text { CDen }][\text { ADen }]) \\
& N=[\beta-\text { CDen }]_{\text {complexed }} /[\text { ADen }]_{\text {complexed }}
\end{aligned}
$$

The PAA $\beta$-CDen/PAAADddn system contrasts with the other two systems in that $N=1.45$ is consistent with one $\beta$-CDen substituent complexing the adamantyl group of the ADddn substit-

(a)

Time $/ \mathrm{min}$

$-100 \quad 102030405060708090100$

(b)

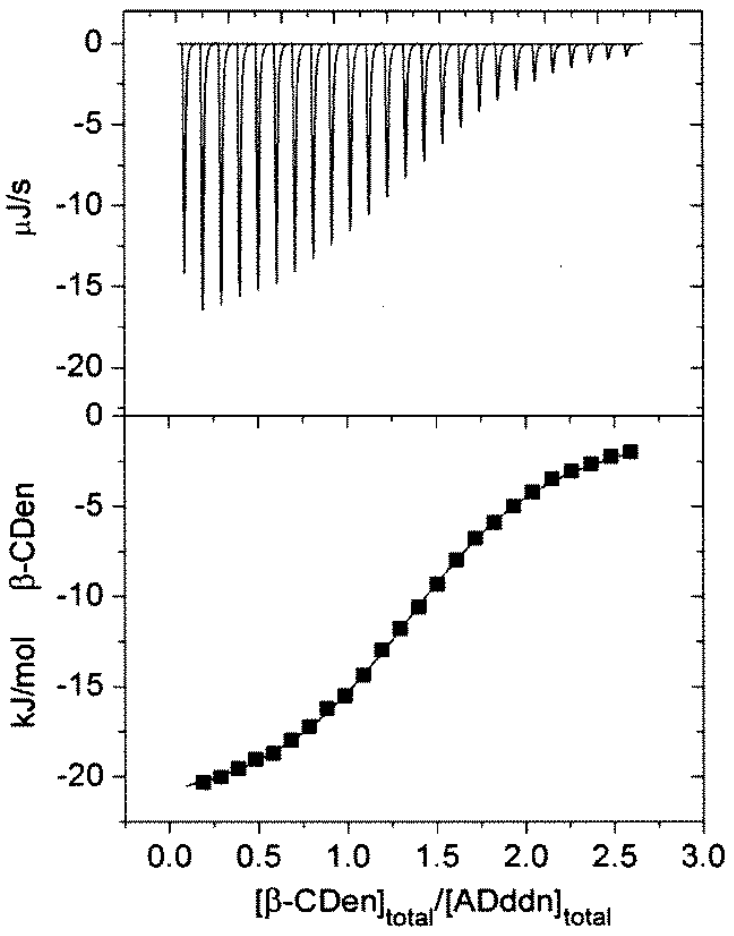

(c)

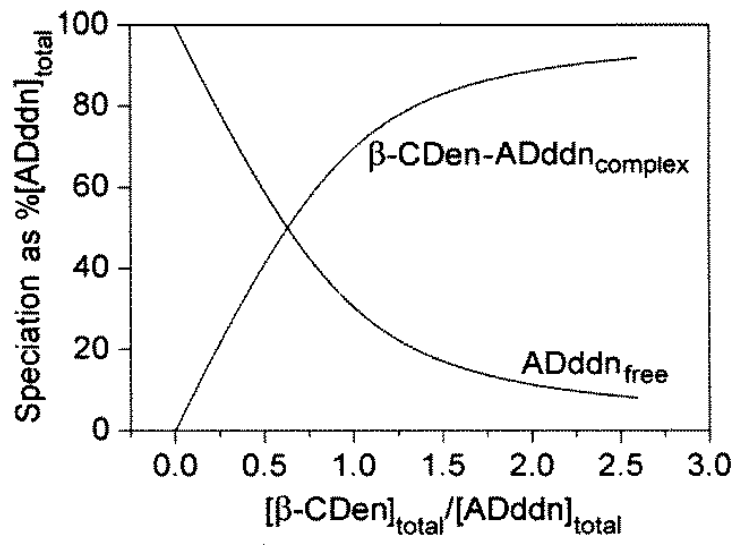

Figure 3: ITC data for the PAA $3-C D e n / P A A A D d d n$ system obtained in aqueous $\mathrm{Na}_{2} \mathrm{HPO}_{4} / \mathrm{KH}_{2} \mathrm{PO}_{4} \mathrm{pH} 7.0$ buffer at $I=0.10 \mathrm{~mol} \mathrm{dm}^{-3}$.

(a) Titration data: for each system $10 \mathrm{~mm}^{3}$ aliquots of a $0.62 \mathrm{wt} \%$ PAA $\beta$-CDen solution $\left([\beta-C D e n]=2.84 \times 10^{-3} \mathrm{~mol} \mathrm{dm}^{-3}\right.$ ) into $1.46 \mathrm{~cm}^{3}$ of 0.064 wt \% PAAADddn ([ADddn] $=1.91 \times 10^{-4} \mathrm{~mol} \mathrm{dm}^{-3}$ ). (b) The solid curve shows the best fit of an algorithm for host-guest complexation between the $\beta-C D e n$ and ADddn substituents to the titration data. (c) Speciation plot showing the variation of [ $\beta-C D e n-A D d d n]_{\text {complex }}$ and of $[A D e n]_{\text {free }}$ as a percentage of $[A D d d n]_{\text {total }}=100 \%$. 


\begin{tabular}{|c|c|c|c|}
\hline Host & & PAA $\beta-C D e n^{b}$ & \\
\hline Guest & PAAADen & PAAADhn & PAAADddn \\
\hline $10^{-3} \mathrm{Kdm}^{3} \mathrm{~mol}^{-1}$ & $28.2 \pm 0.15$ & $28.4 \pm 0.15$ & $39.5 \pm 0.08$ \\
\hline$\Delta H \mathrm{~kJ} \mathrm{~mol}^{-1}$ & $-27.81 \pm 0.55$ & $-25.74 \pm 0.48$ & $-22.36 \pm 0.09$ \\
\hline$T \Delta S \mathrm{~kJ} \mathrm{~mol}^{-1}$ & $-2.42 \pm 0.05$ & $-0.35 \pm 0.01$ & $3.85 \pm 0.08$ \\
\hline$N$ & $0.78 \pm 0.01$ & $0.87 \pm 0.01$ & $1.45 \pm 0.01$ \\
\hline Host & & $\beta-C D^{C}$ & \\
\hline Guest & PAAADen & PAAADhn & PAAADddn \\
\hline $10^{-3} \mathrm{Kdm}^{3} \mathrm{~mol}^{-1}$ & $8.77 \pm 0.24$ & $14.4 \pm 0.06$ & $5.77 \pm 0.18$ \\
\hline$\Delta H \mathrm{~kJ} \mathrm{~mol}^{-1}$ & $-20.81 \pm 0.12$ & $-15.45 \pm 0.09$ & $-16.58 \pm 0.17$ \\
\hline$T \Delta S \mathrm{~kJ} \mathrm{~mol}^{-1}$ & $1.72 \pm 0.18$ & $8.29 \pm 0.18$ & $4.89 \pm 0.24$ \\
\hline$N$ & $0.86 \pm 0.07$ & $0.85 \pm 0.06$ & $0.83 \pm 0.05$ \\
\hline
\end{tabular}

aln aqueous $\mathrm{Na}_{2} \mathrm{HPO}_{4} / \mathrm{KH}_{2} \mathrm{PO}_{4}$ buffer at $\mathrm{pH} 7.0$ and $I=0.10 \mathrm{~mol} \mathrm{dm}{ }^{-3}$. b This study. ${ }^{\mathrm{c}}$ Data from [3] obtained under identical conditions to those of this study. The errors shown are the fitting errors, and the experimental error is $\leq 5 \%$ in both studies.

uent and a second $\beta$-CDen substituent complexing its dodecyl tether in the sequence shown in Figure 4. (Alternatively, the dodecyl tether may be complexed first followed by complexation of the adamantyl group. A further possibility is that the adamantyl group may pass through the annulus of one $\beta$-CDen substituent to be complexed by a second $\beta$-CDen substituent.) The titration curve (Figure 3) could not be resolved into two separate curves consistent with the two complexations being akin to a chelation process which arises from the greater length and flexibility of the dodecyl tether. Ideally, $N=2$ should be observed for such a process, but it appears that either the steric hindrance or hydrophobic association of the adamantyl substituents, or both, restrict $N$ to $<2$. The $2 \mathrm{D}$ NOESY ${ }^{1} \mathrm{H}$ NMR spectrum of the PAA $\beta$-CDen/PAAADddn system confirms the complexation of both the adamantyl group and its dodecyl tether as is discussed below.

The large $K$ for the PAA $\beta$-CDen/PAAADen and PAA $\beta$-CDen/ PAAADhn systems are mainly due to substantial $\Delta H$ contributions (Table 1). The negative $T \Delta S$ for the PAA $\beta-C D e n /$ PAAADen system is attributable to the entropy loss arising from the combination of the $\beta$-CDen and ADen substituents into a single complex and network formation outweighing the entropy gain $[49,50]$ anticipated for the displacement of water from the $\beta$-CDen annulus by the ADen substituent. This offset is smaller for the PAA $\beta$-CDen/PAAADhn system possibly because the ADhn substituent hexyl tether allows more network flexibility. The $K$ for the PAA $\beta$-CDen/PAAADddn system is the largest observed as a consequence of a smaller $\Delta H$ being offset by a positive $T \Delta S$. This probably arises from the entropy loss expected for complexation of the adamantyl group and the dodecyl tether of the ADddn substituent and network formation being outweighed by the entropy gain arising from displacement of water from the $\beta$-CDen annulus by the adamantyl group of ADddn and its dodecyl tether (under the titration conditions the solutions remain fluid whereas at higher concentrations the solution viscosity increases as the network formation becomes more extensive as discussed in the Rheological studies section).

A comparison with the complexation parameters for the $\beta-\mathrm{CD} /$ PAAADen, $\beta-C D / P A A A D h n$ and $\beta-C D / P A A A D d d n$ systems from the literature (Table 1) shows these systems to be characterized by significantly smaller $K$ and $\Delta H$ and more positive $T \Delta S$ [3]. (The $N=0.86-0.83$ are also consistent with either the steric hindrance by the poly(acrylate) backbone, or the hydrophobic association of the adamantyl substituents, or both, hindering complexation). The greater stabilities of the PAA $\beta$ CDen/PAAADen and PAA $\beta$-CDen/PAAADhn systems are attributable to the cooperative stabilizing effect of network formation facilitated by $\beta$-CDen, ADen and ADhn being substituents on the poly(acrylate) backbone, and an accompanying decrease in entropy. The $N=1.45$ and 0.83 (Table 1) for the PAA $\beta$-CDen/PAAADddn and $\beta$-CD/PAAADddn systems, respectively, indicate the greater effect of the dodecyl tether on complexation in the first system by comparison with the second system where some complexation of the dodecyl tether occurs as indicated by ${ }^{1} \mathrm{H}$ NMR spectroscopy but does not result in significant network formation [14].

\section{${ }^{1} \mathrm{H}$ NMR studies of substituted poly(acrylate) network formation}

Further insight into the complexation process is gained from the 2D NOESY ${ }^{1} \mathrm{H}$ NMR spectrum of a $\mathrm{D}_{2} \mathrm{O}$ solution of PAA $\beta$ CDen and PAAADen in which the $\beta$-CDen and ADen substitu- 


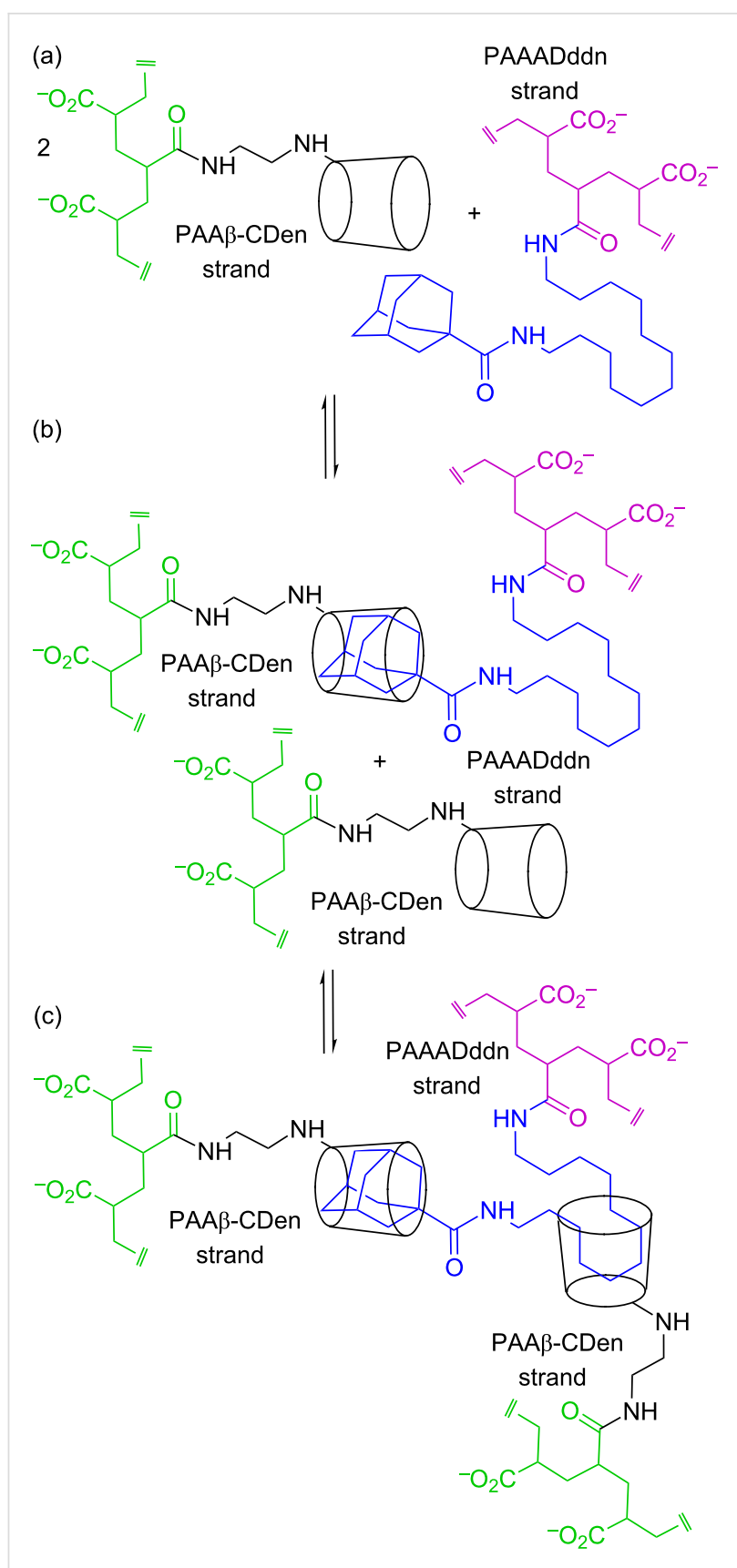

Figure 4: Representation of ditopic complexation of an ADddn substituent of PAAADddn, by two $\beta$-CDen substituents of PAA $\beta$-CDen through initial complexation of the adamantyl group followed by complexation of the dodecyl linker in the sequence (a) to (b) to (c).

ents are equimolar (Figure 5). Substitution of $\beta-\mathrm{CD}$ at $\mathrm{C} 6^{\mathrm{A}}$, as in PAA $\beta$-CDen, renders all of the D-glucopyranose subunits inequivalent such that distinction between the $\mathrm{H} 2-6$ resonances is not possible as a consequence of small differences in the chemical shifts of the resonances of each D-glucopyranose subunit which results in a loss of definition in the $\beta$-CDen substituent spectrum. Thus, the cross-peaks in box A in Figure 5 cannot be unequivocally assigned to the anticipated dominant

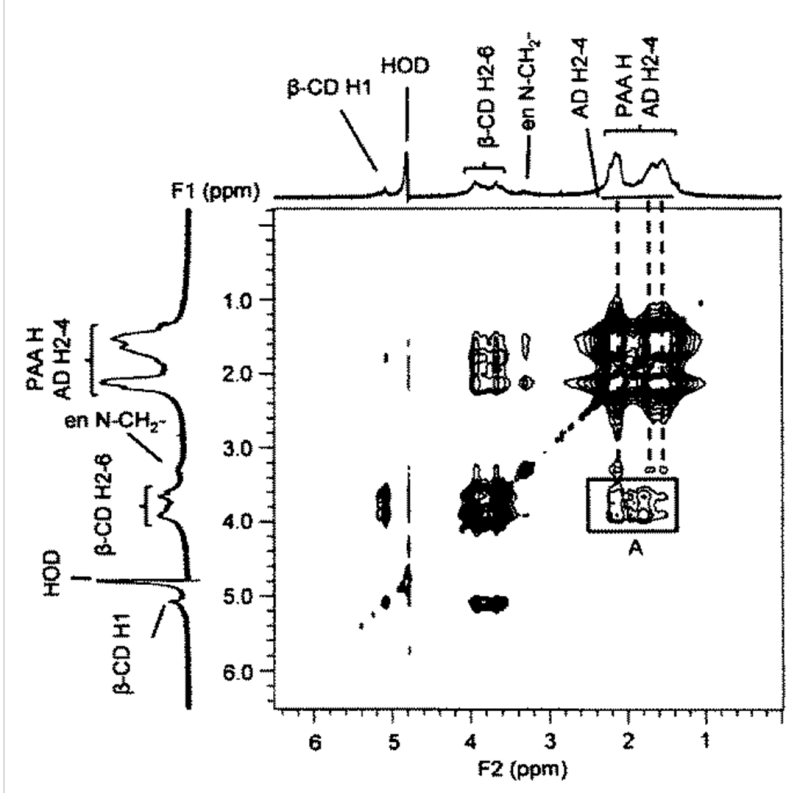

Figure 5: 2D NOESY ${ }^{1} \mathrm{H}$ NMR spectrum of $0.44 \mathrm{wt} \% \mathrm{PAA} \beta-\mathrm{CDen}$ ( $[\beta-$ CDen $]=2.0 \times 10^{-3} \mathrm{~mol} \mathrm{dm}^{-3}$ ) and $0.60 \mathrm{wt} \%$ PAAADen ([ADen] $=$ $2.0 \times 10^{-3} \mathrm{~mol} \mathrm{dm}^{-3}$ ) in $\mathrm{D}_{2} \mathrm{O} \mathrm{Na}{ }_{2} \mathrm{HPO}_{4} / \mathrm{KH}_{2} \mathrm{PO}_{4}$ buffer at $\mathrm{pD} 7.0$ and $I=0.10 \mathrm{~mol} \mathrm{dm}^{-3}$ at $298.2 \mathrm{~K}$. The cross-peaks in box $\mathrm{A}$ are attributed to dipolar interactions between the annular $\mathrm{H} 3,5,6$ protons of the $\beta-C D$ of the $\beta$-CDen substituents and the $\mathrm{H} 2-4$ protons of the ADen substituents.

dipolar interactions between the $\beta$-CDen annular $\mathrm{H} 3,5,6$ protons and the H2-4 ADen protons of the host-guest complex, but they are consistent with such an interaction. The analogous spectrum for the PAA $\beta$-CDen/PAAADhn system (Figure S2, Supporting Information File 1) also shows cross-peaks attributable to dipolar interactions between the $\beta$-CDen annular H3,5,6 protons and the $\mathrm{H} 2-4 \mathrm{ADhn}$ protons. (The 2D NOESY ${ }^{1} \mathrm{H}$ NMR spectrum of PAA $\beta$-CDen alone (Figure S3, Supporting Information File 1) shows no cross-peaks in the region where those assigned to $\beta$-CDen/ADen substituent dipolar interactions arise (Figure 5) indicating that $\beta$-CDen substituent proton dipolar interactions with PAA $\beta$-CDen backbone protons are insignificant.) The similarity of the spectra in Figure 5 and Figure S2 (Supporting Information File 1) and the $K, \Delta H, T \Delta S$ and $N$ data (Table 1 ) for the PAA $\beta$-CDen/PAAADen and PAA $\beta$-CDen/PAAADhn systems is consistent with their host-guest interactions being similar in nature.

The 2D NOESY ${ }^{1} \mathrm{H}$ NMR spectrum of a $\mathrm{D}_{2} \mathrm{O}$ solution of PAA $\beta$-CDen and PAAADddn in which the $\beta$-CDen and ADddn substituents are equimolar shows cross-peaks arising from dipolar interactions between the annular $\mathrm{H} 3,5,6$ protons of the $\beta$-CDen substituent and also those of the H2-4 ADddn protons and the methylene protons of its dodecyl tether (Figure S4, Supporting Information File 1). This is consistent with complexation of both the adamantyl group and the dodecyl tether of the 
ADddn substituent by the $\beta$-CDen substituent, and thereby the likelihood of simultaneous complexation by two $\beta$-CDen substituents as previously discussed. (It has been reported that NOESY ${ }^{1} \mathrm{H}$ NMR cross-peaks between the $\beta$-CD annular $\mathrm{H} 3, \mathrm{H} 5, \mathrm{H} 6$ annular protons and both the $\mathrm{H} 2-4$ ADddn protons and the methylene protons of its dodecyl tether are also observed for solutions of $\beta-\mathrm{CD}$ and PAAADddn [14].)

\section{UV-vis dye complexation studies}

The complexations of the three dyes by $\beta-\mathrm{CD}$ alone in aqueous solution are used as a basis for assessing the effects of the substitution of $\beta$-CDen onto poly(acrylate) in PAA $\beta$-CDen and the subsequent network formation with PAAADen, PAAADhn and PAAADddn on dye complexation. The variation of absorbance of all three dyes with added $\beta-\mathrm{CD}$ is consistent with the dominant formation of 1:1 $\beta$-CD-dye complexes (Equation 4 and Equation 5 where $A, \varepsilon_{\text {dye }}$ and $\varepsilon_{\beta-C D-d y e}$ represent the observed absorbance and the molar absorbance of the dye and the $\beta$-CD-dye complex, respectively) as seen for EO, MO and MR in Figures S5-S7 (Supporting Information File 1), respectively. The wavelengths at which the maximum absorbances occur for the three dyes in their free and complexed states, $\lambda_{\max }$, and the derived $K_{11}$ (Table 2) were determined by best-fitting an algorithm derived from Equations 4-6 to the titration absorbance data using a nonlinear least-squares program, HypSpec [51,52]. The largest and smallest $K_{11}$ characterize the $\beta$-CD-EO and $\beta$-CD-MR host-guest complexes, respectively, and probably reflect the favorable stereochemistry of EO for complexation and its greater hydrophobicity.

$$
\begin{gathered}
\beta-C D+\text { dye } \stackrel{K_{11}}{=} \beta-C D-\text { dye } \\
K_{11}=[\beta-C D-\text { dye }] /([\beta-C D][\text { dye }]) \\
A=\varepsilon_{\text {dye }}[\text { dye }]+\varepsilon_{\beta-C D-d y e}[\beta-C D-\text { dye }]
\end{gathered}
$$

Systematic UV-vis absorbance changes also occur for the dyes upon addition of PAA $\beta$-CDen as seen for EO in Figure 6 and MO and MR in Figures S8 and S9 (Supporting Information File 1), respectively. The $K_{11}$, determined through an algorithm derived from equations analogous to Equations 4-6 in which $\beta-C D$ is replaced by the $\beta$-CDen substituent, are substantially decreased in magnitude by comparison with those derived in the presence of $\beta-C D$, particularly for MR (Table 2). This is attributable to a combination of steric hindrance caused by the

\begin{tabular}{|c|c|c|c|}
\hline Host & Dye & Dye $\lambda_{\max }[\mathrm{nm}]$ & $K_{11}{ }^{a}\left[\mathrm{dm}^{3} \mathrm{~mol}^{-1}\right]$ \\
\hline \multirow[t]{3}{*}{ None } & MR & 430 & - \\
\hline & MO & 464 & - \\
\hline & EO & 474 & - \\
\hline \multirow[t]{3}{*}{$\beta-C D$} & MR & 414 & $772 \pm 10$ \\
\hline & MO & 455 & $3255 \pm 35$ \\
\hline & EO & 466 & $10515 \pm 110$ \\
\hline \multirow[t]{3}{*}{ PAA $\beta-C D e n$} & MR & 415 & $76 \pm 1$ \\
\hline & MO & 458 & $1454 \pm 20$ \\
\hline & EO & 463 & $2230 \pm 30$ \\
\hline \multirow[t]{3}{*}{ PAA $\beta$-CDen/PAAADen } & MR & - & - \\
\hline & MO & 457 & $1000 \pm 10$ \\
\hline & EO & 463 & $1475 \pm 20$ \\
\hline \multirow[t]{3}{*}{ PAA $\beta$-CDen/PAAADhn } & MR & - & - \\
\hline & MO & 457 & $875 \pm 10$ \\
\hline & EO & 463 & $1411 \pm 20$ \\
\hline \multirow[t]{3}{*}{ PAA $\beta$-CDen/PAAADddn } & MR & - & - \\
\hline & $\mathrm{MO}$ & 457 & $713 \pm 10$ \\
\hline & EO & 467 & $986 \pm 20$ \\
\hline
\end{tabular}
poly(acrylate) backbone and repulsion between the PAA $\beta$ CDen carboxylate groups and the negatively charged dyes.

aln aqueous $\mathrm{Na}_{2} \mathrm{HPO}_{4} / \mathrm{KH}_{2} \mathrm{PO}_{4}$ buffer at $\mathrm{pH} 7.0$ and $I=0.10 \mathrm{~mol} \mathrm{dm}{ }^{-3}$ at $298.2 \mathrm{~K}$. The errors shown are fitting errors. The experimental error is $\leq 5 \%$. 

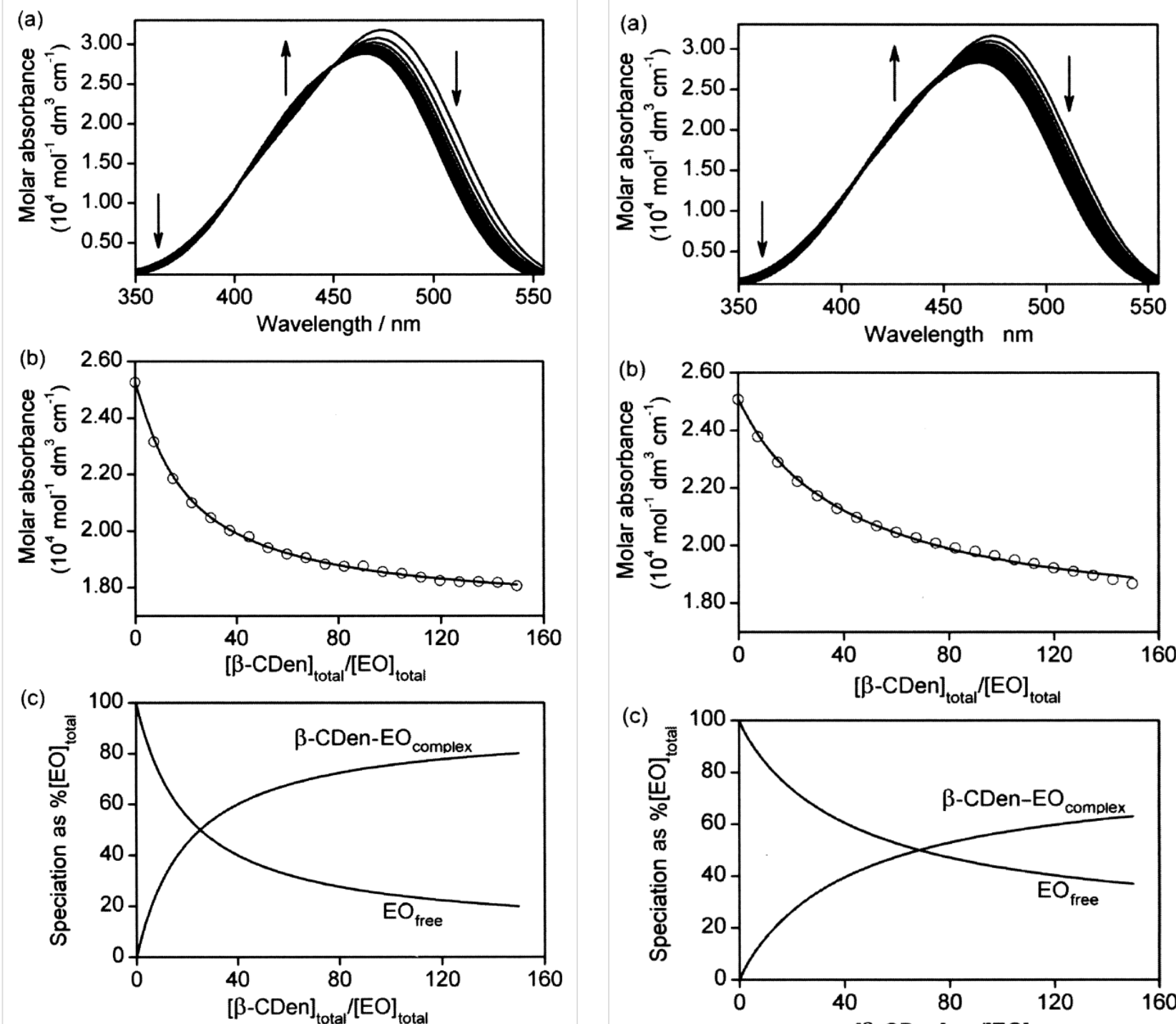

(c)

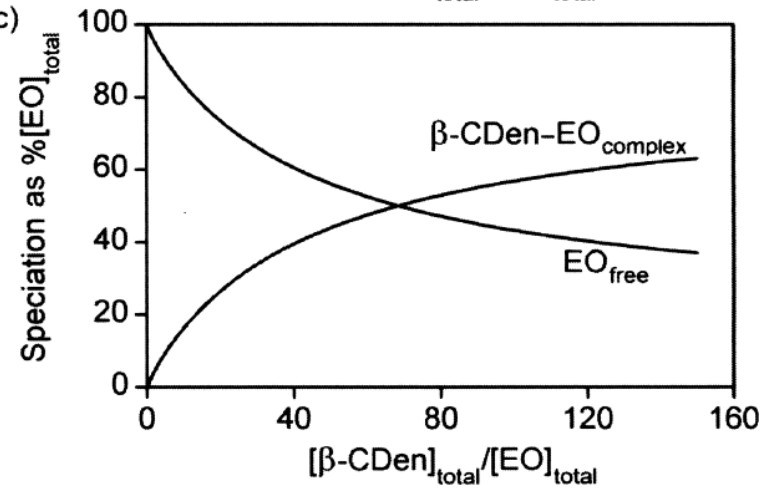

Figure 6: (a) Molar absorbance variation of $1.5 \mathrm{~cm}^{3}$ of a EO solution $\left([E O]=2.00 \times 10^{-5} \mathrm{~mol} \mathrm{dm}^{-3}\right.$ ) with 20 sequential additions of a PAA $\beta$ CDen solution ( $50 \mathrm{~mm}^{3}$ each, $0.98 \mathrm{wt} \%$, $[\beta$-CDen] $=4.49 \times$ $10^{-3} \mathrm{~mol} \mathrm{dm}^{-3}$ ) at $298.2 \mathrm{~K}$. Both solutions were prepared in aqueous $\mathrm{Na}_{2} \mathrm{HPO}_{4} / \mathrm{KH}_{2} \mathrm{PO}_{4}$ buffer at $\mathrm{pH} 7.0$ and $I=0.10 \mathrm{~mol} \mathrm{dm}^{-3}$. The arrows indicate the direction of molar absorbance variation upon each addition of PAA $\beta$-CDen solution. (b) Molar absorbance variation at $500 \mathrm{~nm}$ and the line representing the best fit of an algorithm for a $1: 1$ host-guest complexation of EO by $\beta$-CDen substituents of PAA $\beta$ CDen over the wavelength range $475-525 \mathrm{~nm}$. (c) Speciation plot with $[E O]_{\text {total }}=100 \%$ as the ratio $[\beta-C D e n]_{\text {total }} /[E O]_{\text {total }}$ increases.

The UV-vis variations observed for titration of the dyes with PAA $\beta$-CDen and PAAADen, PAAADhn, or PAAADddn, as exemplified by the ternary PAA $\beta$-CDen/PAAADhn/EO system (Figure 7), reflect the competition between the dye and the adamantyl substituent groups (and also the dodecyl tether for PAAADddn) and the dye for complexation by the $\beta$-CDen substituents of PAA $\beta$-CDen, as do the derived $K_{11}$ (Table 2). Thus, there are two competing equilibria for complexation by the $\beta$-CDen substituent in the PAA $\beta$-CDen/PAAADhn network as

Figure 7: (a) Molar absorbance variation of $1.5 \mathrm{~cm}^{3}$ of a EO solution $\left([E O]=2.00 \times 10^{-5} \mathrm{~mol} \mathrm{dm}^{-3}\right)$ with 20 sequential additions of a PAA $\beta$ CDen solution $\left(25 \mathrm{~mm}^{3}\right.$ each, $1.93 \mathrm{wt} \%$, [ $\beta$-CDen $]=$

$\left.9.03 \times 10^{-3} \mathrm{~mol} \mathrm{dm}^{-3}\right)$ and a PAAADhn solution $\left(25 \mathrm{~mm}^{3}\right.$ each, $0.98 \mathrm{wt} \%,[\mathrm{ADhn}]=3.00 \times 10^{-3} \mathrm{~mol} \mathrm{dm}^{-3}$ ) at $298.2 \mathrm{~K}$. All solutions were prepared in aqueous $\mathrm{Na}_{2} \mathrm{HPO}_{4} / \mathrm{KH}_{2} \mathrm{PO}_{4}$ buffer solutions at $\mathrm{pH} 7.0$ and $I=0.10 \mathrm{~mol} \mathrm{dm}^{-3}$. The arrows indicate the direction of molar absorbance variation upon each addition of the PAA $\beta-C D e n$ and PAAADhn solutions. (b) Molar absorbance variation at $500 \mathrm{~nm}$ and the line representing the best-fit of an algorithm for a 1:1 host-guest complexation of EO by $\beta$-CDen substituents in the self-assembled PAA $\beta$ CDen/PAAADhn network over the wavelength range $475-525 \mathrm{~nm}$. (c) Speciation plot with $[\mathrm{EO}]_{\text {total }}=100 \%$ as the ratio $[\beta-C D e n]_{\text {total }} /[E O]_{\text {total }}$ increases.

shown in Equation 1 and Equation 7. The $K_{11}$ for the host-guest complexation between the $\beta$-CDen substituents in the selfassembled PAA $\beta$-CDen/PAAADhn network and the dye is defined by Equation 8 where [ $\beta$-CDen], [dye] and $[\beta$-CDen-dye] represent the concentration of the $\beta$-CDen substituents in the PAA $\beta$-CDen/PAAADhn network, the dye, and 
the dye complex at equilibrium, respectively. Given that $[\beta-C D e n]_{\text {total }}$ and $[\text { dye }]_{\text {total }}$ are the total concentrations; mass balances are given by Equation 9 and Equation 10. The UV-vis absorbance at a particular wavelength is given by Equation 11 where $A, \varepsilon_{\text {dye }}$ and $\varepsilon_{\mathrm{PAA} \beta} \beta$-CDen-dye represent the observed absorbance and molar absorbance of the dye and the host-guest complex, respectively. The $K_{11}$ (Table 2) were derived by bestfitting an algorithm based on Equations $1-3$ and 7-11 to the UV-vis absorbance data using the HypSpec protocol [51,52].

$$
\begin{gathered}
\beta-\text { CDen }+ \text { dye } \stackrel{K_{11}}{=} \beta-\text { CDen-dye } \\
K_{11}=[\beta-C D e n-d y e] /([\beta-C D h n][\text { dye }]) \\
{[\beta-C D e n]_{\text {total }}=[\beta-C D e n]+[\beta-C D e n-A D h n]} \\
+[\beta-C D e n-d y e] \\
{[\text { dye }]_{\text {total }}=[\text { dye }]+[\beta-C D e n-d y e]} \\
A=\varepsilon_{\text {dye }}[\text { dye }]+\varepsilon_{\text {PAA } \beta-C D e n-d y e}[\beta-C D e n-d y e]
\end{gathered}
$$

The variation of the $\beta$-CDen-EO complex concentration in the PAA $\beta$-CDen/PAAADhn/EO network occurring during the course of titration is shown in Figure 7, and the accompanying changes in free $\beta$-CDen, $\beta$-CDen-ADhn complex and $\beta$-CDen-EO complex concentrations are shown in Figure 8 .

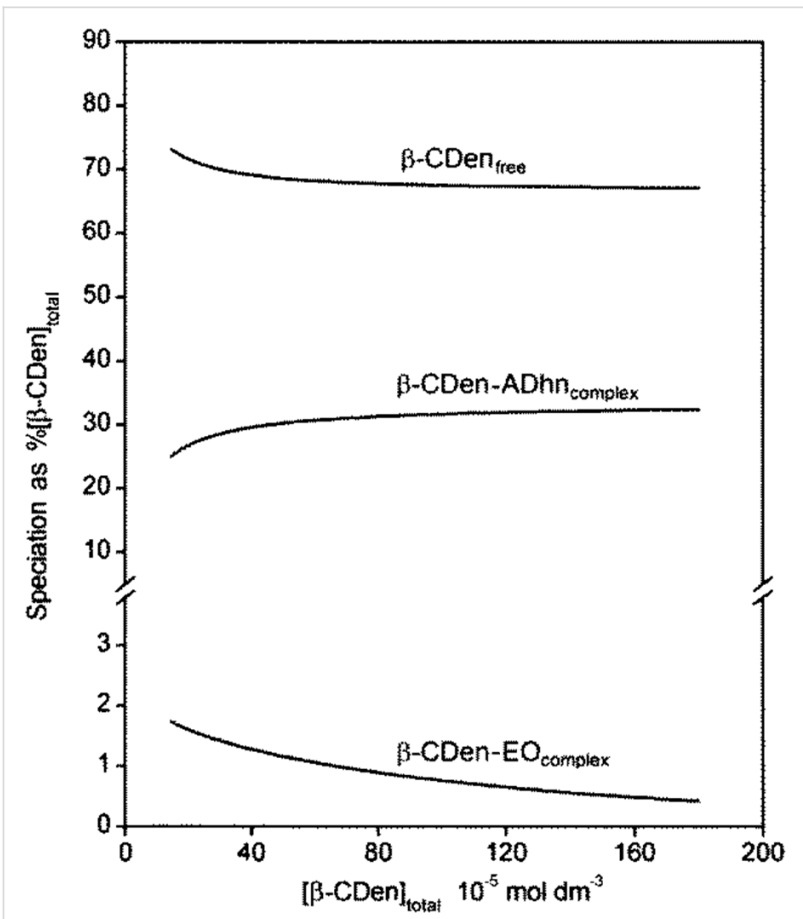

Figure 8: Speciation plot with $[\beta-C D e n]_{\text {total }}=100 \%$ for the $\mathrm{PAA} \beta-$ CDen/PAAADhn/EO system.
The analogous data for PAA $\beta$-CDen/PAAADen/EO are quite similar (Figures S10 and S11, Supporting Information File 1) whereas those for PAA $\beta$-CDen/PAAADddn/EO (Figures S12 and S13, Supporting Information File 1) differ considerably. The corresponding data for the PAA $\beta-\mathrm{CDen} / \mathrm{PAAADen} / \mathrm{MO}$, PAA $\beta$-CDen/PAAADhn/MO and PAA $\beta$-CDen/PAAADddn/ MO systems appear in Figures S14-S19 (Supporting Information File 1). Collectively, these data facilitate determination of $K_{11}$ for these six systems (Table 2) from which it is seen that in each case $K_{11}$ is further decreased by comparison with that determined for complexation by $\beta-\mathrm{CD}$ and PAA $\beta$-CDen, probably because of increased steric crowding within the network. The largest decreases in $K_{11}$ occur for the PAA $\beta-C D e n /$ PAAADddn/MO and PAA $\beta$-CDen/PAAADddn/EO systems; decreases which may reflect the additional competition between the ADddn substituent dodecyl tether and the dyes for complexation by the $\beta$-CDen substituents. (The UV-vis absorbance changes observed for MR in the three networks (Figures S20-S22, Supporting Information File 1) are too small for derivation of $K_{11}$ consistent with further decreases in $K_{11}$ as also observed for the complexation of EO and MO in the analogous systems.)

Equimolar $\mathrm{D}_{2} \mathrm{O}$ solutions of $\beta-\mathrm{CD}$ and each of the three dyes show 2D ${ }^{1} \mathrm{H}$ ROESY NMR cross-peaks arising from dipolar interactions between the $\beta$-CD annular $\mathrm{H} 3,5,6$ protons and those of the dye (Figures S23-S25, Supporting Information File 1) consistent with dye complexation. The $2 \mathrm{D}{ }^{1} \mathrm{H}$ NOESY NMR spectra of solutions of PAA $\beta-C D e n / d y e$, PAA $\beta-C D e n /$ PAAADen/dye, PAA $\beta$-CDen/PAAADhn/dye or PAA $\beta$-CDen/ PAAADddn/dye, where the $\beta$-CDen concentration is $3.6 \times 10^{-3} \mathrm{~mol} \mathrm{dm}^{-3}$, the adamantyl substituent concentration is $1.2 \times 10^{-3} \mathrm{~mol} \mathrm{dm}^{-3}$ in the last three systems and the dye concentration is $2.0 \times 10^{-3} \mathrm{~mol} \mathrm{dm}^{-3}$ show cross-peaks arising from dipolar interactions between the $\beta$-CDen annular H3,5,6 protons and those of the dye, as seen for the PAA $\beta$-CDen/ PAAADen/MR system in Figure 9 (analogous cross-peaks occur in the spectra for the other eleven systems as shown in Figures S26-S36, Supporting Information File 1), consistent with dye complexation in all twelve systems. Thus, despite the $K_{11}$ for complexation of MR by the PAA $\beta$-CDen/PAAADen, PAA $\beta$-CDen/PAAADhn and PAA $\beta$-CDen/PAAADddn networks being too small for reliable determination in the UV-vis studies, the observation of significant cross-peaks arising from dipolar interactions between the $\beta$-CDen substituent annular H3,5,6 protons and the MR $\mathrm{Ha}-\mathrm{g}$ protons indicate the occurrence of complexation.

\section{Rheological studies}

At higher solution concentrations than those studied by UV-vis spectroscopy, the networks formed by the three combinations: 


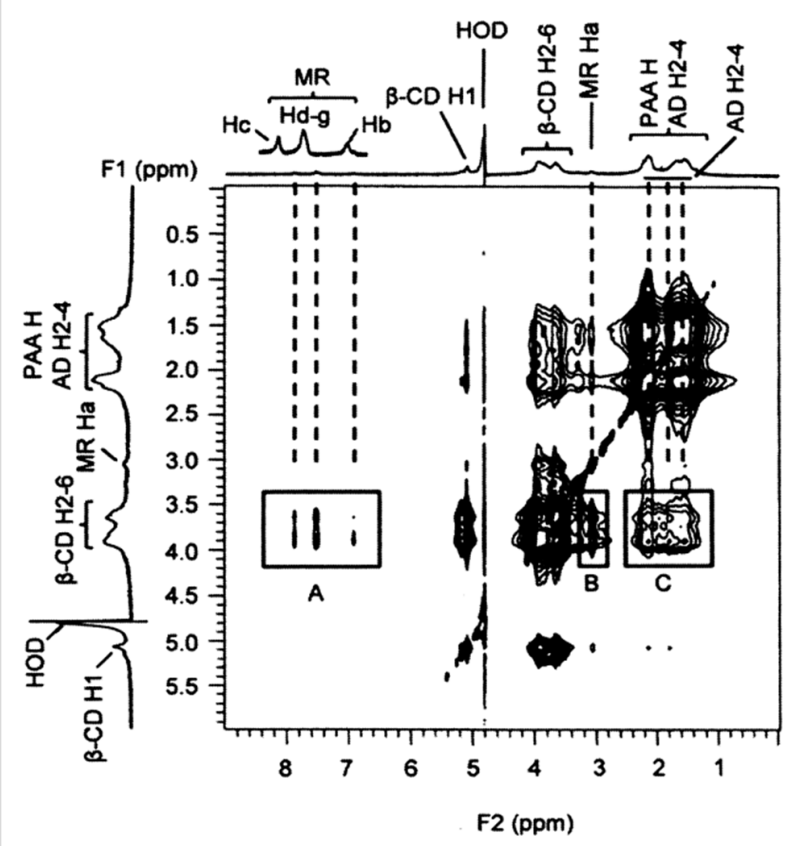

Figure 9: $2 \mathrm{D}$ NOESY ${ }^{1} \mathrm{H}$ NMR spectrum of $M R([M R]=$ $\left.2.0 \times 10^{-3} \mathrm{~mol} \mathrm{dm}^{-3}\right)$ in solution with PAA $\beta$-CDen $(0.78 \mathrm{wt} \%$, $\left.[\beta-C D e n]=3.6 \times 10^{-3} \mathrm{~mol} \mathrm{dm}^{-3}\right)$ and PAAADen $(0.40 \mathrm{wt} \%$, $[$ ADen $]=$ $1.2 \times 10^{-3} \mathrm{~mol} \mathrm{dm}^{-3}$ ) in $\mathrm{D}_{2} \mathrm{O} \mathrm{Na} \mathrm{HPO}_{4} / \mathrm{KH}_{2} \mathrm{PO}_{4}$ buffer solution at pD 7.0 and $I=0.10 \mathrm{~mol} \mathrm{dm}^{-3}$ at $298.2 \mathrm{~K}$. Cross-peaks in boxes $A$ and $B$ are attributed to dipolar interactions of the annular $\mathrm{H} 3,5,6$ protons of $\beta$-CDen and the aromatic $(\mathrm{Hb}-\mathrm{g})$ and methyl $(\mathrm{Ha})$ protons of MR, respectively. Cross-peaks in box $\mathrm{C}$ arise from dipolar interactions between the annular $\mathrm{H} 3,5,6$ protons of $\beta-\mathrm{CDen}$ and the $\mathrm{H} 2-4$ protons of ADen.

PAA $\beta$-CDen/PAAADen, PAA $\beta$-CDen/PAAADhn and PAA $\beta$ CDen/PAAADddn separately and when complexing MR, MO or EO form hydrogels, the viscosities of which were determined by rheology. In each hydrogel [ $\beta$-CDen] $=$ $3.60 \times 10^{-3} \mathrm{~mol} \mathrm{dm}^{-3}$ and [ADen, ADhn or ADddn] $=$ $1.20 \times 10^{-3} \mathrm{~mol} \mathrm{dm}^{-3}$, the concentration of each dye was $2.00 \times 10^{-3} \mathrm{~mol} \mathrm{dm}^{-3}$ and the overall concentration of substituted poly(acrylate)s was 1.14-1.20 wt \%. (These hydrogel compositions are identical to those used in the dye release studies discussed below, and are presented in Table S1, Supporting Information File 1.) The viscosity variation of each system with the shear rate is shown for the binary systems PAA $\beta$-CD/PAAADen, PAA $\beta-C D / P A A A D h n$ and PAA $\beta-C D /$ PAAADddn in Figure S37 (Supporting Information File 1), and for the ternary systems in which each of the binary systems complexes the three dyes in Figure S38, Supporting Information File 1). The viscosities show small variations in the shear rate and those determined at $0.03 \mathrm{~s}^{-1}$ shear rate are selected for comparison purposes. (Because it was necessary to quantitatively determine the rates of dye release from these hydrogels, their fluidities must be sufficient to allow their quantitative transfer into the dye release measurement apparatus (Figure S39, Sup- porting Information File 1), and this determined their maximum component concentrations.)

The viscosity variations at a $0.03 \mathrm{~s}^{-1}$ shear rate for all twelve systems are shown in Figure 10 from which it is seen that, in the absence of dyes, the viscosities of the binary systems increase in the sequence: $1.14 \mathrm{w} \%$ PAA $\beta$-CDen/PAAADen (1.44 Pa s) $<1.18$ wt \% 1.20 wt \% PAA $\beta$-CDen/PAAADhn $(1.75 \mathrm{~Pa} \mathrm{~s})<$ PAA $\beta-C D e n / P A A A D d d n$ (4.85 Pa s). Thus, it appears that the two-fold $\beta$-CDen complexation of both the adamantyl group and the dodecyl tether of the ADddn substituent in the PAA $\beta$ CDen/PAAADddn system (Figure 4), deduced to be present from the ITC and 2D NOESY ${ }^{1} \mathrm{H}$ NMR studies, increases the viscosity and extent of network formation of the $1.20 \mathrm{wt} \%$ solutions of this system by comparison with those of the PAA $\beta$ CDen/PAAADen and PAA $\beta-C D e n / P A A A D h n$ systems.

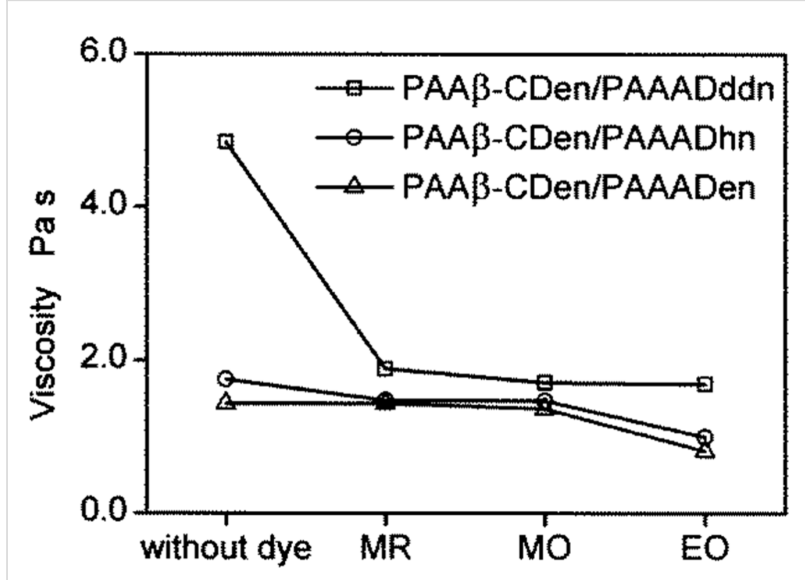

Figure 10: Viscosity variations at a $0.03 \mathrm{~s}^{-1}$ shear rate of $1.14 \mathrm{wt} \%$ PAA $\beta$-CDen/PAAADen, 1.18 wt \% PAA $\beta$-CDen/PAAADhn, or 1.20 wt $\%$ PAA $\beta-C D e n / P A A A D d d n$ in the absence and presence of $\mathrm{MR}, \mathrm{MO}$, or $\mathrm{EO}$ at $298.2 \mathrm{~K}$ in aqueous $\mathrm{Na}_{2} \mathrm{HPO}_{4} / \mathrm{KH}_{2} \mathrm{PO}_{4}$ buffer at $\mathrm{pH} 7.0$ and $I=0.10 \mathrm{~mol} \mathrm{dm}^{-3}$. In each system, the concentrations of the $\beta$-CDen and adamantyl substituents were $3.60 \times 10^{-3} \mathrm{~mol} \mathrm{dm}^{-3}$ and $1.20 \times 10^{-3} \mathrm{~mol} \mathrm{dm}^{-3}$, respectively, and the dye concentration was $2.00 \times 10^{-3} \mathrm{~mol} \mathrm{dm}^{-3}$.

Upon addition of MR, MO or EO, a substantial decrease in the viscosities of the ternary PAA $\beta$-CDen/PAAADddn/dye solutions occurs (viscosities $=4.85,1.89,1.71$ and $1.59 \mathrm{~Pa} \mathrm{~s}$ in the presence of no dye, MR, MO and EO, respectively) whereas the changes in the $0.03 \mathrm{~s}^{-1}$ shear rate viscosities of the PAA $\beta$ CDen/PAAADen $(1.44,1.49,1.37$ and $0.87 \mathrm{~Pa}$ s in the presence of no dye, MR, MO and EO, respectively) and PAA $\beta$ CDen/PAAADhn $(1.75,1.48,1.53$ and $1.00 \mathrm{~Pa}$ s in the presence of no dye, MR, MO and EO, respectively) solutions are smaller. This is consistent with the dye complexation competing with that of the dodecyl tether of the ADddn substituent for a second $\beta$-CDen substituent in the PAA $\beta$-CDen/PAAADddn/dye systems, even with the $\beta$-CDen substituent concentration being 
1.8 times greater than that of the dyes, whereas this option is not available in the PAA $\beta$-CDen/PAAADen/dye and PAA $\beta-C D e n /$ PAAADhn/dye systems. Nevertheless, the $0.03 \mathrm{~s}^{-1}$ shear rate viscosities of the PAA $\beta$-CDen/PAAADddn/dye systems are still greater than those of the PAA $\beta$-CDen/PAAADen/dye and PAA $\beta$-CDen/PAAADhn/dye systems consistent with some residual complexation of the dodecyl tether of the ADddn substituent and a consequent viscosity enhancement.

The viscosities of the PAA $\beta$-CDen/PAAADen/dye and PAA $\beta$ CDen/PAAADhn/dye systems show little variation in the presence of MR and MO, but a small decrease occurs in the presence of EO consistent with it showing the largest $K_{11}$ for both systems (as determined in the UV-vis studies) and competing more strongly with ADen and ADhn for complexation by $\beta$-CDen. The general picture which emerges for dye complex-

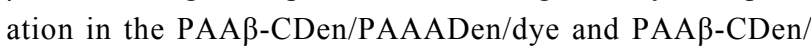
PAAADhn/dye systems is shown in Figure 11. In the PAA $\beta$ CDen/PAAADddn/dye systems the dodecyl tether of the ADddn substituent is complexed by the $\beta$-CDen substituent as shown by $2 \mathrm{D}{ }^{1} \mathrm{H}$ NOESY NMR (Figures S34-S36, Supporting Information File 1) consistent with it competing with the dyes for complexation by $\beta$-CDen.

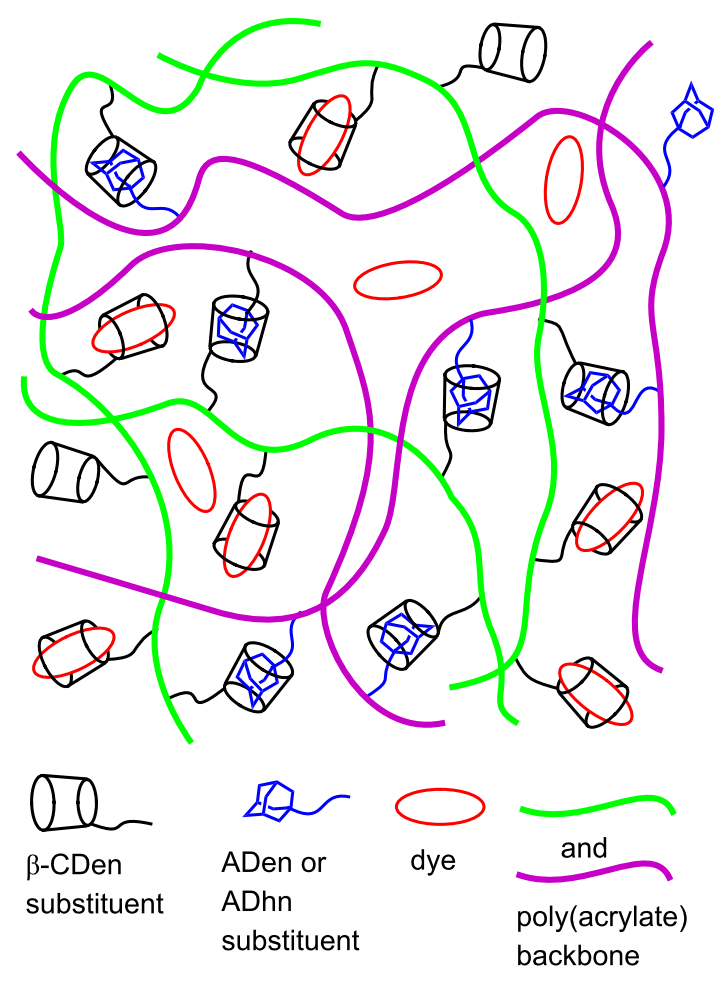

Figure 11: Representation of dye complexation in the PAA $3-C D e n /$ PAAADen and PAA $\beta-C D e n / P A A A D h n$ networks.

\section{Dye release studies}

Dye release through a dialysis membrane with pores allowing passage of species with a molecular weight up to $3.5 \mathrm{kDa}$ into an aqueous $\mathrm{Na}_{2} \mathrm{HPO}_{4} / \mathrm{KH}_{2} \mathrm{PO}_{4}$ buffer at $\mathrm{pH} 7.0$, $I=0.10 \mathrm{~mol} \mathrm{dm}^{-3}$ and $298.2 \mathrm{~K}$ was characterized for each system. Reference solutions of MR, MO and EO were prepared in aqueous $\mathrm{Na}_{2} \mathrm{HPO}_{4} / \mathrm{KH}_{2} \mathrm{PO}_{4}$ buffer at $\mathrm{pH} 7.0$ and $I=0.10 \mathrm{~mol} \mathrm{dm}^{-3}$. All other dye solutions were prepared in the same buffer. To render the dye environment as similar as possible with respect to poly(acrylate), PAA, backbone concentration, those solutions containing neither PAA $\beta-C D e n /$ PAAADen, PAA $\beta-C D e n / P A A A D h n$ nor PAA $\beta-C D e n /$ PAAADddn had an appropriate amount of PAA added. The compositions of the thirty-six solutions studied appear in the caption to Figure 12 and in more detail in Table S1 (Supporting Information File 1.

The profiles of dye released with time for EO, MR and MO shown in Figure 12 exhibit informative trends. The solutions of EO in (a) $\mathrm{Na}_{2} \mathrm{HPO}_{4} / \mathrm{KH}_{2} \mathrm{PO}_{4}$ buffer alone, (b) $1.20 \mathrm{wt} \% \mathrm{PAA}$, (e) $1.14 \mathrm{wt} \%$ PAA/PAAADen, (d) $1.18 \mathrm{wt} \%$ PAA/PAAADhn and (c) $1.20 \mathrm{wt} \%$ PAA/PAAADddn show very similar release profiles (Figure 12(i)) with $90 \%$ of EO released within $20 \mathrm{~h}$ indicative of little interaction between EO and the other solutes. This is consistent with the major factors determining the appearance of EO in the receiving solution being its diffusion within the particular EO sample and its interaction with the dialysis membrane as it passes through its pores. Similar profiles characterize the release of MO and MR from PAA and adamantyl substituted PAA (Figure 12(ii) and 12(iii), respectively,) and a similar interpretation applies. However, the release of EO from a solution of (f) $1.20 \mathrm{wt} \% \mathrm{PAA} / \beta-\mathrm{CD}$ is slower reaching $87 \%$ after $48 \mathrm{~h}$ consistent with substantial formation of the $\beta$-CD-EO complex which, although of lower molecular weight than the membrane $3.5 \mathrm{kDa}$ limit, is likely to pass through the membrane less readily than EO alone and is less mobile than free EO. An even slower release of EO from the (j) 1.18 wt \% PAA/PAA $\beta$-CDen solution occurs reaching only $69 \%$ after $48 \mathrm{~h}$ consistent with substantial formation of the $\beta$-CDen-EO complex within the PAA $\beta-C D e n / E O$ solution which is of too high molecular weight to pass through the membrane such that the passage is limited to free EO alone. These data are consistent with complexation of EO by either $\beta-C D$ alone or a $\beta$-CDen substituent within PAA $\beta$-CDen controlling the amount of free EO in solution and thereby the rate of release through the membrane. A similar slowing of release is seen for the analogous MO and MR solutions (Figure 12(ii) and 12(iii)).

The EO release rate profiles for the (i) $1.14 \mathrm{wt} \%$ PAA $\beta$-CDen/ PAAADen, h) $1.18 \mathrm{wt} \%$ PAA $\beta$-CDen/PAAADhn and (g) $1.20 \mathrm{wt} \%$ PAA $\beta$-CDen/PAAADddn networks fall between 


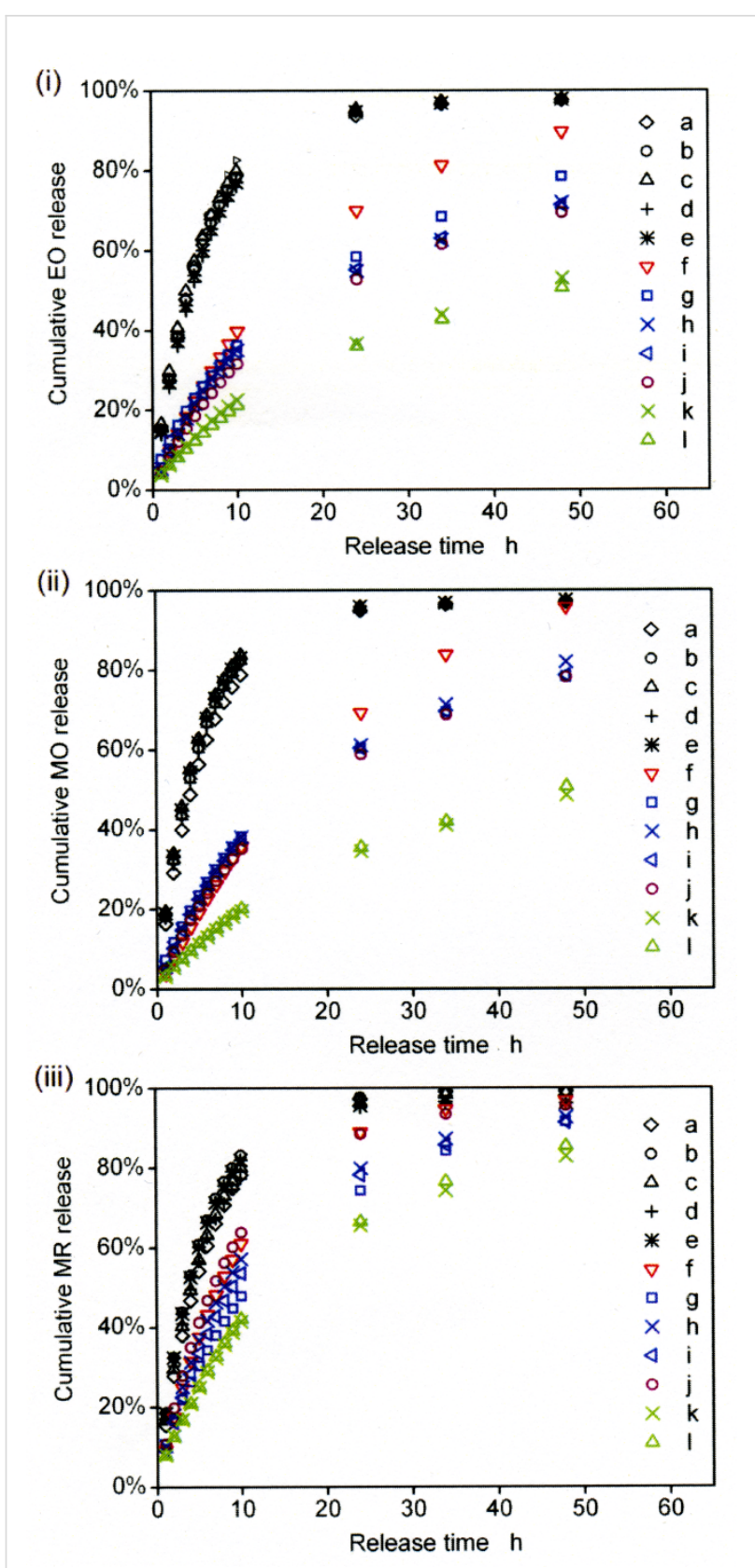

Figure 12: Release profiles for $E O$ (i), $M O$ (ii) and MR (iii) from aqueous (a) $\mathrm{Na}_{2} \mathrm{HPO}_{4} / \mathrm{KH}_{2} \mathrm{PO}_{4}$ buffer alone, (b) $1.20 \mathrm{wt} \% \mathrm{PAA}$ (c) $1.20 \mathrm{wt} \%$ PAA/PAAADddn, (d) $1.18 \mathrm{wt} \%$ PAA/PAAADhn, (e) 1.14 wt \% PAA/PAAADen, (f) 1.20 wt \% PAA/ $\beta-C D$, (g) 1.20 wt \% PAA $\beta-C D e n / P A A A D d d n,(h) 1.18$ wt \% PAAß-CDen/PAAADhn, (i) 1.14 wt \% PAA $\beta-C D e n / P A A A D e n$, (j) $1.18 w t \%$ PAA/PAA $3-C D e n$, (k) 1.96 wt \% PAAB-CDen/PAAADhn, and (I) 1.89 wt \% PAAß-CDen/ PAAADen all in aqueous $\mathrm{Na}_{2} \mathrm{HPO}_{4} / \mathrm{KH}_{2} \mathrm{PO}_{4}$ buffer at $\mathrm{pH} 7.0$, $I=0.10 \mathrm{~mol} \mathrm{dm}^{-3}$ and $298.2 \mathrm{~K}$. Initial [dye] $=2.00 \times 10^{-3} \mathrm{~mol} \mathrm{dm}^{-3}$.

those for the (f) $1.20 \mathrm{wt} \% \mathrm{PAA} / \beta-\mathrm{CD}$ and (j) $1.18 \mathrm{wt} \% \mathrm{PAA} /$ PAA $\beta$-CDen solutions consistent with EO complexation by the $\beta$-CDen substituents being of major importance in controlling the rate of EO release. The release of EO is significantly more rapid from the $(\mathrm{g}) 1.20 \mathrm{wt} \%$ PAA $\beta$-CDen/PAAADddn solu- tion than from the (h) $1.18 \mathrm{wt} \%$ PAA $\beta$-CDen/PAAADhn and (i) $1.14 \mathrm{wt} \% \mathrm{PAA} \beta-\mathrm{CDen} / \mathrm{PAAADen}$ solutions, but this variation is much less for MR and MO (Figure 12(ii) and 12(iii)). This coincides with the (g) 1.20 wt \% PAA $\beta$-CDen/ PAAADddn/EO solution being substantially more viscous than the (h) $1.18 \mathrm{wt} \%$ PAA $\beta-C D e n / P A A A D h n / E O$ and (i) $1.14 \mathrm{wt} \% \mathrm{PAA} \beta-\mathrm{CDen} / \mathrm{PAAADen} / \mathrm{EO}$ solutions whereas the viscosities of the three corresponding MR and MO solutions are more similar (Figure 10). This indicates that viscosity is a significant rate determining factor for dye release.

The effect of increasing network extension and viscosity on the rate of EO release is illustrated by the profiles for the more viscous (1) $1.89 \mathrm{wt} \%$ PAA $\beta$-CDen/PAAADen and (k) $1.96 \mathrm{wt} \% \mathrm{PAA} \beta-\mathrm{CDen} / \mathrm{PAAADhn}$ solutions from which $50 \%$ of EO is released in $48 \mathrm{~h}$, and is attributable to an increase in $\beta$-CDen substituent concentration increasing the proportion of EO complexed and the more extensive network slowing free EO movement. A slowing of dye release for the analogous MO and MR solutions (Figure 12(ii) and 12(iii), respectively) is similarly explained. (A $1.90 \mathrm{wt} \%$ PAA $\beta$-CDen/PAAADddn solution was too viscous to transfer quantitatively to the release apparatus.)

While the release of MR in (a) $\mathrm{Na}_{2} \mathrm{HPO}_{4} / \mathrm{KH}_{2} \mathrm{PO}_{4}$ buffer alone, (b) 1.20 wt \% PAA, (e) $1.14 \mathrm{wt} \%$ PAA/PAAADen, (d) $1.18 \mathrm{wt} \%$ PAA/PAAADhn and (c) $1.20 \mathrm{wt} \%$ PAA/ PAAADddn solutions (Figure 12(iii)) show profiles similar to those observed for EO (Figure 12(i)), the profiles for MR release from the (f) $1.20 \mathrm{wt} \% \mathrm{PAA} / \beta-\mathrm{CD}$ solution and the six solutions, (g)-(1), containing PAA $\beta$-CDen are compressed into a shorter time-frame of more rapid release than that observed for the corresponding EO solutions. This is consistent with the weaker complexing of MR by comparison with EO (Table 2) and the higher proportion of free MR facilitating more rapid release. The release profiles for MO (Figure 12(ii)) more closely resemble those of EO as anticipated from the strength of complexing of MO being between that of EO and MR (Table 2).

The release rates of the three dyes from their (j) $1.18 \mathrm{wt} \%$ PAA/PAA $\beta-C D e n / d y e$, (i) 1.14 wt \% PAA $\beta$-CDen/PAAADen/ dye and (h) 1.18 wt \% PAA $\beta$-CDen/PAAADhn/dye solutions decrease in the order MR $>$ MO $>$ EO (Figures S40-S42, Supporting Information File 1) consistent with the same order of decrease in $K_{11}$ for dye complexation (Table 2) largely determining the relative release rates. While the release of MR from the (g) $1.20 \mathrm{wt} \%$ PAA $\beta$-CDen/PAAADddn/dye solution is the most rapid, the release profiles of MO and EO are more similar (Figure S43, Supporting Information File 1). This indicates that the dodecyl tether of the ADddn substituent diminishes the 
effect of the magnitude of $K_{11}$ on the relative release rates of $\mathrm{MO}$ and EO possibly as a result of variations in the network structure as MO and EO compete with the dodecyl tether for complexation in the $\beta$-CDen substituent annuli. Finally, while the rate of MR release from the (f) $1.20 \mathrm{wt} \% \mathrm{PAA} / \beta-\mathrm{CD} /$ dye solution (Figure S44, Supporting Information File 1) is the most rapid, the two analogous but less rapid release profiles for MO and EO overlap which probably reflects a combination of the abilities of MO, EO and the $\beta-\mathrm{CD}-\mathrm{MO}$ and $\beta-\mathrm{CD}-\mathrm{EO}$ complexes to pass through the pores of the dialysis membrane.

\section{Conclusion}

The self-assembly of the $\beta$-CDen and ADen, ADhn and ADddn substituted poly(acrylate) networks, PAA $\beta$-CDen/PAAADen, PAA $\beta$-CDen/PAAADhn and PAA $\beta$-CDen/PAAADddn, and their complexation and release of the dyes, ethyl orange, methyl orange and methyl red have been characterized in aqueous solution. The factors controlling the dye release from these networks are the strength of complexation of the dye, which is dependent upon the structure of the dye, and the structure of the network and its viscosity. Potentially, these systems form the basis for the development of controlled drug delivery systems for topical and wound applications, where the factors for drug release are likely to be similar to those controlling the dye release.

\section{Experimental Materials}

The sodium salts of methyl red (BDH), methyl orange (BDH), ethyl orange (Sigma-Aldrich), and $\beta$-cyclodextrin (Nihon Shokuhin Kako Co.) were used as received. Poly(acrylic acid) $\left(M_{\mathrm{W}}=250 \mathrm{kDa}, M_{\mathrm{W}} / M_{\mathrm{n}} \approx 2\right)$ was purchased from Aldrich as a $35 \mathrm{wt} \%$ aqueous solution and freeze-dried to a constant weight. The substituted poly(acrylate)s: PAA $\beta$-CDen [15], PAAADen [13], PAAADhn [17] and PAAADddn [17] (Figure 1) were synthesized according to literature procedures, and the extent of random substitution of poly(acrylate) with the $\beta$-CDen, ADen, ADhn and ADddn substituents was determined from their ${ }^{1} \mathrm{H}$ NMR spectra to be $8.8 \pm 0.2 \%, 3.3 \pm 0.1 \%, 3.0 \pm 0.1 \%$ and $2.9 \pm 0.1 \%$, respectively.

\section{NMR spectroscopy}

A Varian Inova 600 spectrometer operating at $599.96 \mathrm{MHz}$ was used to run 1D, 2D NOESY and $2 \mathrm{D}$ ROESY ${ }^{1} \mathrm{H}$ NMR spectra using standard pulse sequences with a mixing time of $0.3 \mathrm{~s}$ in the last two cases. All sample solutions were prepared in $\mathrm{D}_{2} \mathrm{O}$ $\mathrm{Na}_{2} \mathrm{HPO}_{4} / \mathrm{KH}_{2} \mathrm{PO}_{4}$ buffer solutions at $\mathrm{pD} 7.0$ and $I=0.10 \mathrm{~mol} \mathrm{dm}^{-3}$ and were equilibrated at the thermostated probe temperature of $298.2 \pm 0.1 \mathrm{~K}$ for $30 \mathrm{~min}$ in $5 \mathrm{~mm}$ diameter NMR tubes prior to their spectra being recorded. Chemical shifts $(\delta, \mathrm{ppm})$ were internally referenced to HOD at $\delta=4.79 \mathrm{ppm}$. The substitution percentage of the $\beta$-CDen substituents on the PAA $\beta$-CDen backbone was determined from a comparison of the resonance areas of the $\beta-\mathrm{CD} H 1$ protons and the poly(acrylate) protons. The substitution percentages of the adamantyl substituents on the PAAADen, PAAADhn and PAAADddn backbones were determined through a comparison of the resonance area of the methylene protons of both $-\mathrm{NHCH}_{2}$ tether groups with that of the superimposed resonance areas of the poly(acrylate), adamantyl H2-4 and other tether methylene protons. The compositions of all solutions appear in the corresponding figure captions.

\section{Isothermal titration calorimetry (ITC)}

ITC measurements were made using a MicroCal VP isothermal titration calorimeter. In each titration, $10 \mathrm{~mm}^{3}$ aliquots of a $0.62 \mathrm{wt} \%$ PAA $\beta$-CDen solution $\left([\beta-\mathrm{CDen}]=2.84 \times 10^{-3} \mathrm{~mol}\right.$ $\mathrm{dm}^{-3}$ ) were titrated into $1.46 \mathrm{~cm}^{3}$ of either a $0.062 \mathrm{wt} \%$ PAAADen $\left([\right.$ ADen $\left.]=2.06 \times 10^{-4} \mathrm{~mol} \mathrm{dm}^{-3}\right), 0.064 \mathrm{wt} \%$ PAAADhn $\left([\mathrm{ADhn}]=1.91 \times 10^{-4} \mathrm{~mol} \mathrm{dm}^{-3}\right)$, or $0.072 \mathrm{wt} \%$ PAAADddn $\left([\right.$ ADddn $\left.]=2.03 \times 10^{-4} \mathrm{~mol} \mathrm{dm}^{-3}\right)$ solution at $298.2 \mathrm{~K}$ using a computer-controlled micro-syringe at intervals of $210 \mathrm{~s}$. All solutions were prepared in aqueous $\mathrm{Na}_{2} \mathrm{HPO}_{4} / \mathrm{KH}_{2} \mathrm{PO}_{4}$ buffer at $\mathrm{pH} 7.0$ and $I=0.10 \mathrm{~mol} \mathrm{dm}^{-3}$. The heats of dilution were determined by titrating aqueous $\mathrm{Na}_{2} \mathrm{HPO}_{4} / \mathrm{KH}_{2} \mathrm{PO}_{4}$ buffer ( $\mathrm{pH} 7.0$ and $I=0.10 \mathrm{~mol} \mathrm{dm}^{-3}$ ) into similarly buffered PAAADen, PAAADhn and PAAADddn solutions and by titrating similarly buffered PAA $\beta$-CDen solution into the buffer solution. The heats of dilution were subtracted from the total heats evolved to give the heats of host-guest complexation from which the complexation constant, $K$, and the corresponding $\Delta H, T \Delta S$ and $N$ were calculated using the Origin 7.0 MicroCal protocol [53] as described in the Results and Discussion section.

\section{UV-vis spectroscopy}

The UV-vis spectra were recorded with a Cary-Varian 5000 $\mathrm{UV}$-vis spectrophotometer using $1 \mathrm{~cm}$ path length matched quartz cells. All UV-vis titrations were performed in aqueous $\mathrm{Na}_{2} \mathrm{HPO}_{4} / \mathrm{KH}_{2} \mathrm{PO}_{4}$ buffer at $\mathrm{pH} 7.0$ and $I=0.10 \mathrm{~mol} \mathrm{dm}^{-3}$ at $298.2 \mathrm{~K}$. For the $\beta-\mathrm{CD} /$ dye titrations, $50 \mathrm{~mm}^{3}$ aliquots of a $\beta-C D$ solution were sequentially titrated into $1.5 \mathrm{~cm}^{3}$ of each dye solution and $1.5 \mathrm{~cm}^{3}$ of each reference solution. The UV-vis absorbance spectra were recorded prior to and after each of 20 sequential additions of $\beta-C D$ solution. The PAA $\beta-C D e n / d y e$ titrations where carried out in a similar manner using the same solution volumes. For the PAA $\beta-C D e n /$ adamantyl-substituted PAA/dye studies, a $25 \mathrm{~mm}^{3}$ aliquot of a PAA $\beta$-CDen solution followed by $25 \mathrm{~mm}^{3}$ aliquots of a PAAADen, PAAADhn, or PAAADddn solution were sequentially titrated in a twenty-fold sequence into $1.5 \mathrm{~cm}^{3}$ of each dye solution and $1.5 \mathrm{~cm}^{3}$ of each reference solution. The UV-vis 
absorbance spectra were recorded prior to and after each of the twenty sequential additions of PAA $\beta$-CDen solution and a PAAADen, PAAADhn, or PAAADddn solution. The concentrations of all solutions used in the UV-vis titrations are given in the figure captions. The complexation constants for dye complexation, $K_{11}$, and the corresponding $\Delta H$ and $T \Delta S$ were derived from the UV-vis data as described in the Results and Discussion section.

\section{Rheology}

Rheological measurements were carried out with a Physica MCR 501 (Anton Parr GmbH) stress-controlled rheometer with a $25 \mathrm{~mm}$ cone and plate geometry. The temperature was controlled at $298.2 \mathrm{~K}$ by a Peltier plate. All solutions were prepared in aqueous $\mathrm{Na}_{2} \mathrm{HPO}_{4} / \mathrm{KH}_{2} \mathrm{PO}_{4}$ buffer at $\mathrm{pH} 7.0$ and $I=0.10 \mathrm{~mol} \mathrm{dm}^{-3}$. The compositions of sample solutions are shown in the corresponding figure captions.

\section{Dye release}

Dye release studies were performed using a membrane diffusion apparatus (Figure S37, Supporting Information File 1) in which a $3.5 \mathrm{kDa}$ molecular weight cut-off dialysis membrane (Spectr/Por 3) of surface area $7.0 \mathrm{~cm}^{2}$ separated $5.0 \mathrm{~cm}^{3}$ of the dye containing solution (made up in $\mathrm{Na}_{2} \mathrm{HPO}_{4} / \mathrm{KH}_{2} \mathrm{PO}_{4}$ buffer at $\mathrm{pH} 7.0$ and $I=0.10 \mathrm{~mol} \mathrm{dm}^{-3}$ with MR, MO or EO concentration $=2.00 \times 10^{-3} \mathrm{~mol} \mathrm{dm}^{-3}$ ) from $200 \mathrm{~cm}^{3}$ of aqueous $\mathrm{Na}_{2} \mathrm{HPO}_{4} / \mathrm{KH}_{2} \mathrm{PO}_{4}$ buffer at $\mathrm{pH} 7.0$ and $I=0.10 \mathrm{~mol} \mathrm{dm}^{-3}$ which acted as the receiving solution. During the release experiments, the receiving solution was stirred at 298.2 K. At appropriate time intervals, a $2.0 \mathrm{~cm}^{3}$ sample of the receptor solution was withdrawn, its UV-vis spectrum was measured and the sample was then returned to the receiving solution. The dye concentration in the receiving solution was calculated by reference to its molar absorbance spectrum determined under the same conditions. Each dye solution composition is given in Table S1 (Supporting Information File 1). All solutions were stirred and equilibrated at $298.2 \mathrm{~K}$ before dye release measurements commenced.

\section{Supporting Information}

\section{Supporting Information File 1}

Additional titrations, spectra and data.

[http://www.beilstein-journals.org/bjoc/content/

supplementary/1860-5397-13-183-S1.pdf]

\section{Acknowledgements}

We gratefully acknowledge the support of this research by the Australian Research Council through Discovery Project DP110103177 (SFL and CJE) and by the National Science
Foundation of China through Grants 20774028 and 20774030 $(\mathrm{XG})$.

\section{References}

1. Lecourt, T.; Sinay, P.; Chassenieux, C.; Rinaudo, M.; Auzély-Velty, R. Macromolecules 2004, 37, 4635-4642. doi:10.1021/ma049822x

2. Wenz, G. Adv. Polym. Sci. 2009, 222, 204-254. doi:10.1007/12_2008_13

3. Nguyen, H.-T.; Pham, D.-T.; Lincoln, S. F.; Wang, J.; Guo, X.; Easton, C. J.; Prud'homme, R. K. Polym. Chem. 2013, 4, 820-829. doi:10.1039/C2PY20746J

4. Wang, J.; Xu, Y.; Wang, Y.; Liu, J.; Xu, J.; Li, L.; Nguyen, H.-T.; Pham, D.-T.; Lincoln, S. F.; Guo, X. RSC Adv. 2015, 5, 46067-46073. doi:10.1039/C5RA06163F

5. Auzély-Velty, R.; Rinaudo, M. Macromolecules 2002, 35, 7955-7962. doi:10.1021/ma020664o

6. Guo, X.; Abdala, A. A.; May, B. L.; Lincoln, S. F.; Khan, S. A.; Prud'homme, R. K. Macromolecules 2005, 38, 3037-3040. doi:10.1021/ma050071o

7. Guo, X.; Abdala, A. A.; May, B. L.; Lincoln, S. F.; Khan, S. A.; Prud'homme, R. K. Polymer 2006, 47, 2976-2983. doi:10.1016/j.polymer.2006.03.006

8. Charlot, A.; Auzély-Velty, R. Macromolecules 2007, 40, 1147-1158. doi:10.1021/ma062322e

9. Semenov, A.; Charlot, A.; Auzély-Velty, R.; Rinaudo, M. Rheol. Acta 2007, 46, 541-568. doi:10.1007/s00397-006-0149-4

10. Koopmans, C.; Ritter, H. Macromolecules 2008, 41, 7418-7422. doi:10.1021/ma801202f

11. Li, L.; Guo, X.; Wang, J.; Liu, P.; Prud'homme, R. K.; May, B. L.; Lincoln, S. F. Macromolecules 2008, 41, 8677-8681. doi:10.1021/ma8020147

12. Li, L.; Guo, X.; Fu, L.; Prud'homme, R. K.; Lincoln, S. F. Langmuir 2008, 24, 8290-8296. doi:10.1021/la800859w

13. Wang, J.; Li, L.; Ke, H.; Liu, P.; Zheng, L.; Guo, X.; Lincoln, S. F. Asia-Pac. J. Chem. Eng. 2009, 4, 537-543. doi:10.1002/apj.279

14. Guo, X.; Wang, J.; Li, L.; Pham, D.-T.; Clements, P.; Lincoln, S. F.; May, B. L.; Chen, Q.; Zheng, L.; Prud'homme, R. K. J. Polym. Sci., Part B: Polym. Phys. 2010, 48, 1818-1825. doi:10.1002/polb.22052

15. Guo, X.; Wang, J.; Li, L.; Pham, D.-T.; Clements, P.; Lincoln, S. F.; May, B. L.; Chen, Q.; Zheng, L.; Prud'homme, R. K. Macromol. Rapid Commun. 2010, 31, 300-304. doi:10.1002/marc.200900560

16. Stadermann, J.; Komber, H.; Erber, M.; Däbritz, F.; Ritter, H.; Voit, B. Macromolecules 2011, 44, 3250-3259. doi:10.1021/ma200048a

17. Wang, J.; Pham, D.-T.; Kee, T. W.; Clafton, S. N.; Guo, X.; Clements, P.; Lincoln, S. F.; Prud'homme, R. K.; Easton, C. J. Macromolecules 2011, 44, 9782-9791. doi:10.1021/ma202055a

18. Pham, D.-T.; Nguyen, H.-T.; Lincoln, S. F.; Wang, J.; Guo, X.; Easton, C. J.; Prud'homme, R. K. J. Polym. Sci., Part A: Polym. Chem. 2015, 53, 1278-1286. doi:10.1002/pola.27563

19. Wang, J.; Qu, Z.; Wang, Y.; Li, L.; Guo, X.; Pham, D.-T.; Lincoln, S. F.; Prud'homme, R. K. Beilstein J. Org. Chem. 2016, 12, 50-72. doi:10.3762/bjoc. 12.7

20. Hirayama, F.; Uekama, K. Adv. Drug Delivery Rev. 1999, 36, 125-141. doi:10.1016/S0169-409X(98)00058-1

21. Bibby, D. C.; Davies, N. M.; Tucker, I. G. Int. J. Pharm. 2000, 197, 1-11. doi:10.1016/S0378-5173(00)00335-5 
22. Davis, M. E.; Brewster, M. E. Nat. Rev. Drug Discovery 2004, 3 , 1023-1035. doi:10.1038/nrd1576

23. Loftsson, T.; Duchêne, D. Int. J. Pharm. 2007, 329, 1-11. doi:10.1016/j.ijpharm.2006.10.044

24. van de Manakkar, F.; Vermonden, T.; van Nostrum, C. F.; Hennink, W. E. Biomacromolecules 2009, 10, 3157-3175. doi:10.1021/bm901065f

25. Layre, A.-M.; Volet, G.; Wintgens, V.; Amiel, C. Biomacromolecules 2009, 10, 3283-3289. doi:10.1021/bm900866p

26. Davis, M. E. Mol. Pharmaceutics 2009, 6, 659-668. doi: $10.1021 / \mathrm{mp} 900015 \mathrm{y}$

27. Thiele, C.; Auerbach, D.; Jung, G.; Qiong, L.; Schneider, M.; Wenz, G. Polym. Chem. 2010, 2, 209-215. doi:10.1039/COPY00241K

28. Gao, W.; Chan, J. M.; Farokhzad, O. C. Mol. Pharmaceutics 2010, 7, 1913-1920. doi:10.1021/mp100253e

29. Maciollek, A.; Munteanu, M.; Ritter, H. Macromol. Chem. Phys. 2010, 211, 245-249. doi:10.1002/macp.200900436

30. Böhm, I.; Kreth, S. K.; Ritter, H. Beilstein J. Org. Chem. 2011, 7, 1130-1134. doi:10.3762/bjoc.7.130

31. Yhaya, F.; Lim, J.; Kim, Y.; Liang, M.; Gregory, A. M.; Stenzel, M. H. Macromolecules 2011, 44, 8433-8445. doi:10.1021/ma2013964

32. Mellet, C. O.; García Fernández, J. M.; Benito, J. M. Chem. Soc. Rev. 2011, 40, 1586-1608. doi:10.1039/C0CS00019A

33. Albuzat, T.; Keil, M.; Ellis, J.; Alexander, C.; Wenz, G. J. Mater. Chem. 2012, 22, 8558-8565. doi:10.1039/c2jm16425f

34. Luo, G.-F.; Xu, X.-D.; Zhang, J.; Yang, J.; Gong, Y.-H.; Lei, Q.; Jia, H.-Z.; Li, C.; Zhuo, R.-X.; Zhang, X.-Z. ACS Appl. Mater. Interfaces 2012, 4, 5317-5324. doi:10.1021/am301258a

35. Trotta, F.; Zanetti, M.; Cavalli, R. Beilstein J. Org. Chem. 2012, 8 , 2091-2099. doi:10.3762/bjoc.8.235

36. Li, Z.; Yin, H.; Zhang, Z.; Liu, K. L.; Li, J. Biomacromolecules 2012, 13, 3162-3172. doi:10.1021/bm300936x

37. Harada, T.; Giorgio, L.; Harris, T. J.; Pham, D.-T.; Ngo, H. T.; Need, E. F.; Coventry, B. J.; Lincoln, S. F.; Easton, C. J.; Buchanan, G.; Kee, T. W. Mol. Pharmaceutics 2013, 10, 4481-4490. doi:10.1021/mp400309s

38. Wang, D.; Xie, D.; Shi, W.; Sun, S.; Zhao, C. Langmuir 2013, 29, 8311-8319. doi:10.1021/la401201w

39. Kanasty, R.; Dorkin, J. R.; Vegas, A.; Anderson, D. Nat. Mater. 2013, 12, 967-977. doi:10.1038/nmat3765

40. Kutyła, M. J.; Lambert, L. K.; Davies, N. M.; McGeary, R. P.; Shaw, P. N.; Ross, B. P. Int. J. Pharm. 2013, 444, 175-184. doi:10.1016/j.ijpharm.2013.01.005

41. Jing, J.; Szarpak-Jankowska, A.; Guillot, R.; Pignot-Paintrand, I.; Picart, C.; Auzély-Velty, R. Chem. Mater. 2013, 25, 3867-3873. doi:10.1021/cm4019925

42. Harada, T.; McTernan, H. L.; Pham, D.-T.; Lincoln, S. F.; Kee, T. W. J. Phys. Chem. B 2015, 119, 2425-2433. doi:10.1021/jp507272f

43. Zhao, Y.; Tian, W.; Yang, G.; Fan, X. Beilstein J. Org. Chem. 2014, 10, 2696-2703. doi:10.3762/bjoc.10.284

44. Li, Y.; Guo, H.; Gan, J.; Zheng, J.; Zhang, Y.; Wu, K.; Lu, M. J. Polym. Res. 2015, 22, 91. doi:10.1007/s10965-015-0720-8

45. Sharker, S. M.; Kim, S. M.; Kim, S. H.; In, I.; Lee, H.; Park, S. Y. J. Mater. Chem. B 2015, 3, 5833-5841. doi:10.1039/C5TB00779H

46. Duchene, D.; Cavalli, R.; Gref, R. Curr. Pharm. Biotechnol. 2016, 17, 248-255. doi:10.2174/1389201017666151030104944

47. Liu, J.; Hennink, W. E.; van Steenbergen, M. J.; Zhuo, R.; Jiang, X. Bioconjugate Chem. 2016, 27, 1143-1152. doi:10.1021/acs.bioconjchem.6b00094
48. Eftink, M. R.; Andy, M. L.; Bystrom, K.; Perlmutter, H. D.; Kristol, D. S. J. Am. Chem. Soc. 1989, 111, 6765-6772. doi:10.1021/ja00199a041

49. Rekharsky, M. V.; Inoue, Y. Chem. Rev. 1998, 98, 1875-1918. doi:10.1021/cr970015o

50. Harries, D.; Rau, D. C.; Parsegian, V. A. J. Am. Chem. Soc. 2005, 127, 2184-2190. doi:10.1021/ja045541t

51. Gans, P.; Sabatini, A.; Vacca, A. Talanta 1996, 43, 1739-1753. doi:10.1016/0039-9140(96)01958-3

52. HypSpec; Protonic Software: 2 Templegate Avenue, Leeds LS15 OHD, UK.

53. MicroCal, 22 Industrial Drive East, Northampton, MA01060, USA.

\section{License and Terms}

This is an Open Access article under the terms of the Creative Commons Attribution License

(http://creativecommons.org/licenses/by/4.0), which permits unrestricted use, distribution, and reproduction in any medium, provided the original work is properly cited.

The license is subject to the Beilstein Journal of Organic Chemistry terms and conditions: (http://www.beilstein-journals.org/bjoc)

The definitive version of this article is the electronic one which can be found at: doi:10.3762/bjoc. 13.183 